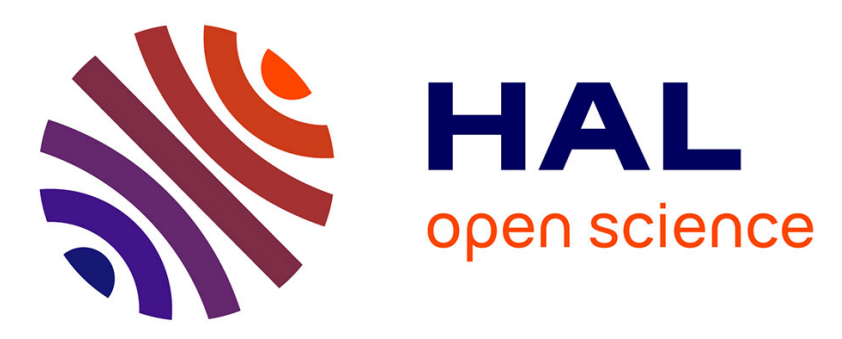

\title{
Simultaneous Seismic Sources Separation Based on Matrioshka Orthogonal Matching Pursuit, Application in Oil and Gas Exploration
}

\author{
Ekaterina Shipilova, Michel Barret, Matthieu Bloch, Jean-Luc Boelle, \\ Jean-Luc Collette
}

\section{To cite this version:}

Ekaterina Shipilova, Michel Barret, Matthieu Bloch, Jean-Luc Boelle, Jean-Luc Collette. Simultaneous Seismic Sources Separation Based on Matrioshka Orthogonal Matching Pursuit, Application in Oil and Gas Exploration. IEEE Transactions on Geoscience and Remote Sensing, 2020, pp.1-18. 10.1109/TGRS.2019.2959650 . hal-02625829

\section{HAL Id: hal-02625829}

https://hal-centralesupelec.archives-ouvertes.fr/hal-02625829

Submitted on 26 May 2020

HAL is a multi-disciplinary open access archive for the deposit and dissemination of scientific research documents, whether they are published or not. The documents may come from teaching and research institutions in France or abroad, or from public or private research centers.
L'archive ouverte pluridisciplinaire HAL, est destinée au dépôt et à la diffusion de documents scientifiques de niveau recherche, publiés ou non, émanant des établissements d'enseignement et de recherche français ou étrangers, des laboratoires publics ou privés. 


\title{
Simultaneous Seismic Sources Separation Based on Matrioshka Orthogonal Matching Pursuit, Application in Oil and Gas Exploration
}

\author{
Ekaterina Shipilova, Michel Barret ${ }^{(}$, Matthieu Bloch ${ }^{\circledR}$, Jean-Luc Boelle, and Jean-Luc Collette
}

\begin{abstract}
We present Matrioshka orthogonal matching pursuit (OMP), a method consisting of two nested OMPs for separating seismic sources at an early stage of the signal processing chain. Matrioshka OMP is based on models of sensor signals that place nonrestrictive assumptions on the seismic survey using simultaneous sources. Our seismic event model is based on the spatial coherence of signals, which results in a straight or slightly curved feature in the trace representation of the data with a specific wavelet, whose magnitude can linearly vary according to the offset. We demonstrate the effectiveness of the approach on synthetic and real data.
\end{abstract}

Index Terms-Acquisition, matching pursuit, optimization methods, seismic signal processing, sources separation.

\section{INTRODUCTION}

\section{A. Simultaneous-Source Seismic Acquisition} (1)

$\mathrm{S}$ EISMIC surveys are performed at all stages of oil and gas exploration and development, with the objective of constructing an image of the subsurface without actually penetrating into the Earth's crust. To obtain such an image, seismic sources generate a wavefield at or close to the surface, which then propagates into the subsurface where it is altered and reflected by the geological layers and bodies. The geological medium only absorbs some of the emitted energy and the remaining energy escapes and reaches the surface, where seismic receivers sensitive to minute vibrations record it. With some assumptions regarding the propagation velocities, the knowledge of the emission and detection time instants, as well as the spatial positions of the sources and receivers, provides information about the subsurface geometry and physical properties.

When simultaneous sources emit their signals, or when a single source emits a long signal or makes small pauses

Manuscript received February 8, 2019; revised July 25, 2019 and November 12, 2019; accepted November 21, 2019. (Corresponding author: Michel Barret.)

Ekaterina Shipilova and Jean-Luc Boelle are with TOTAL, CSTJF, 64018 Pau, France (e-mail: ekaterina.shipilova@total.com; jean-luc.boelle @ total.com).

Michel Barret is with CentraleSupélec, 57070 Metz, France, and also with the UMI 2958 Georgia Tech-CNRS, 57070 Metz, France (e-mail: michel.barret@centralesupelec.fr).

Matthieu Bloch is with the School of Electrical and Computer Engineering, Georgia Institute of Technology, Atlanta, GA 30332 USA, and also with the UMI 2958 Georgia Tech-CNRS, 57070 Metz, France (e-mail: matthieu.bloch@ece.gatech.edu).

Jean-Luc Collette is with CentraleSupélec, 57070 Metz, France (e-mail: jean-luc.collette@centralesupelec.fr).

Color versions of one or more of the figures in this article are available online at http://ieeexplore.iee.org.

Digital Object Identifier 10.1109/TGRS.2019.2959650

between subsequent short shots, one must be able to separate the different sources and the different shots to identify the exact time of emission associated with each seismic event encountered. Since the crosstalk (effect of pollution of one signal by another) between shots significantly complicates the signal processing and eventually degrades image quality [1], conventional seismic surveys ensure that the time and location intervals between shots are large enough to avoid crosstalk. Nevertheless, simultaneous-source seismic data acquisition has recently attracted attention for its potential to acquire larger amounts of data in a reduced time [2], which might be beneficial in harsh meteorological environments [3] or because of environmental regulations.

The idea of allowing multiple seismic sources to fire simultaneously was first introduced in the seventies for marine and land seismic [4], [5], but the first simultaneous shooting was only implemented for land vibroseis acquisition in the late nineties [6] by controlling the pattern of the sources, also known as source sweeping. Since then, vibratory seismic source techniques have constantly improved, and sweep generation and management is still actively ongoing [7]-[9]. The first proposal of simultaneous shooting without constraints on the source pattern, i.e., without specific encoding or sweep management, dates back to the late nineties [10], but actual implementation of appropriate logistics, survey design, and processing has taken nearly a decade. The difficulty may be attributed in part to the dithering of shooting times required for best wavefield separation, which could result in complex real-time communication and synchronization of the sources in the field [11]. It was not until 2006 that BP proposed a new approach called independent simultaneous sourcing (ISS ${ }^{1}$ ), in which no effort is made to synchronize the sources [12], and the burden is placed on the receiver to process a continuous recording. Subsequent published tests on synthetic and real data [13] have established the usefulness and potential of data acquired in simultaneous-source mode [14]; however, these early tests did not exploit any specific processing and only relied on the noise attenuation capacity of stacking for crosstalk suppression. Moreover, subsequent full scale surveys were only held at the exploration stage, in zones where structural interpretation was needed [15]-[18]. To speed up seismic campaigns, industry is now envisioning the use of simultaneous shooting at all exploration and development stages, including those having reservoir characterization [19],

${ }^{1}$ ISS is a registered trade mark of BP p.l.c. 
[20] and monitoring [21]-[24] purposes. Consequently, more sophisticated processing is needed to achieve the high precision necessary at these stages. Seismic processing engineers could also benefit from simultaneous sources separation methods to suppress interference between neighboring seismic surveys [25].

The seismic data resulting from simultaneous-source shooting is now colloquially known as blended data. The methods proposed to process blended seismic data can be classified into the three following groups. Most of them impose constraints on data acquisition, as the firing times of different sources must be random enough, except for some completely different techniques, such as seismic apparition [26] or coherent simultaneous shooting [27].

1) Random Noise Attenuation: These methods consist in presenting the signal coming from each source as being coherent and removing the incoherent signals coming from the other sources using conventional denoising procedures such as median filtering [28]-[30], prediction error filtering [31], or a combination of these filters [32].

2) Inversion-Based Source Separation: These methods treat the signals of each source as signal and not as noise; they aim to explain all interpretable signals present in the data. The inversion approach has been the most successful so far and has been shown to render superior performance over the random noise attenuation [33]. The common mathematical formulation of such methods is given in [34]; this includes a coherency constraint usually expressed in a transform domain rather than the $(t, x)$ domain, e.g., Fourier domain [35]-[37], Radon domain [38], curvelet domain [39], seislet domain [40], or in more sophisticated domain combinations [41] [43]. The method presented in this article falls into this category.

3) Direct Imaging of Blended Data: These methods aim at processing blended data without explicit separation [44]. This approach has the potential to reduce computational complexity, since explicit source separation typically increases the amount of data volumes to process: a separate data set is created for each source.

Direct imaging methods might be the most promising in the future but would require a complete and costly overhaul of the currently used seismic signal processing chain. Since existing state-of-the-art industrial seismic processing algorithms are not compatible with blended data, there is still much interest in deblending the raw seismic signals to keep the subsequent processing unchanged.

Many of the currently proposed deblending methods need some preprocessing of the data, e.g., surface wave suppression [33]. In this article, we propose to use a data-driven seismic event model in a greedy decomposition to obtain a separation suitable for application at the earliest processing stages. We start by briefly recalling in Section II notions of seismics from a perspective that facilitates the description of our method and identifies the conditions required for our method to apply. We then introduce in Section II-C the data-driven parametric model of a seismic event, which includes a curvature parameter and a magnitude attenuation factor depending on the position of the source. We describe in Section III the main contributions of this article, which are the data-driven model and the decomposition method implementation. We also state in Sections II and III the assumptions that are necessary and sufficient to apply our method. Finally, we illustrate in Section IV the performance of our method on synthetic and real seismic data.

\section{B. Signal Decomposition and Orthogonal Matching Pursuit}

Let the signal to decompose be $d(t) \in \mathcal{H}$, where $\mathcal{H}$ is a Hilbert space, with inner product and Euclidean norm, respectively, defined by $\langle d, g\rangle=\int_{-\infty}^{+\infty} d(t) \bar{g}(t) d t$ (where $\bar{g}$ is the complex conjugate of $g$ ) and $\|d\|=\langle d, d\rangle^{1 / 2}$. The inner product becomes $\langle d, g\rangle=\sum_{t \in \Delta_{t} \mathbb{Z}} d(t) \overline{g(t)}$ after sampling with a period $\Delta_{t}$. A dictionary $\mathcal{D}$ is a subset $\left\{g_{\gamma}(t)\right\}_{\gamma \in \Omega} \subset \mathcal{H}$ comprised of unit-norm vectors indexed by a set $\Omega \subset \mathbb{R}^{\nu}$, with $v \in \mathbb{N}$. The elements of a dictionary are called atoms.

When decomposing a signal $d$ into a linear combination of $L \mathcal{D}$-atoms, we look for a subset $\Gamma \subset \Omega$ of $L$ elements and complex numbers $\left\{c_{\gamma}\right\}_{\gamma \in \Gamma}$ that lead to the smallest approximation error $\min _{\{\Gamma \subset \Omega:|\Gamma|=L\}} \min _{\left\{c_{\gamma}\right\}}\left\|d-\sum_{\gamma \in \Gamma} c_{\gamma} g_{\gamma}\right\|$. If we knew $L$ and $\Gamma$ a priori, we could solve this problem with least-squares methods. However, for such complex dictionaries as ours, we cannot fix $L$ and $\Gamma$ beforehand; we first have to choose an optimal set of atoms and then find a linear combination that best approximates the signal.

Greedy algorithms, such as matching pursuit (MP) [45] and orthogonal matching pursuit (OMP) [46], provide an efficient solution to that problem. It consists in constructing successive approximations of $d$ by making orthogonal projections on elements of $\mathcal{D}$. Let us set $R^{0} d=d$ and suppose that the $(\ell-1)$ th order residue $R^{\ell-1} d$ is computed for $\ell \geq 1$. Then, $R^{\ell-1} d$ is decomposed into $R^{\ell-1} d=\left\langle R^{\ell-1} d, g_{\gamma_{\ell}}\right\rangle g_{\gamma_{\ell}}+R^{\ell} d$. This leads to $\left\langle R^{\ell-1} d, g_{\gamma_{\ell}}\right\rangle=\left\langle R^{\ell-1} d, g_{\gamma_{\ell}}\right\rangle\left\langle g_{\gamma_{\ell}}, g_{\gamma_{\ell}}\right\rangle+\left\langle R^{\ell} d, g_{\gamma_{\ell}}\right\rangle$, which shows that the residue $R^{\ell} d$ is orthogonal to $g_{\gamma \ell}$, since the atoms are unit-norm vectors. Hence, $\left\|R^{\ell-1} d\right\|^{2} \stackrel{\gamma_{\ell}}{=}$ $\left|\left\langle R^{\ell-1} d, g_{\gamma_{\ell}}\right\rangle\right|^{2}+\left\|R^{\ell} d\right\|^{2}$, and to minimize the norm of the residue $\left\|R^{\ell} d\right\|$, one must choose $g_{\gamma_{\ell}} \in \mathcal{D}$ such that

$$
\left|\left\langle R^{\ell-1} d, g_{\gamma_{\ell}}\right\rangle\right|=\max _{\gamma \in \Omega}\left|\left\langle R^{\ell-1} d, g_{\gamma}\right\rangle\right| .
$$

If we now carry the decomposition up to the $L$ th order, we obtain: $d=\sum_{\ell=1}^{L}\left\langle R^{\ell-1} d, g_{\gamma_{\ell}}\right\rangle g_{\gamma_{\ell}}+R^{L} d$ and $\|d\|^{2}=$ $\sum_{\ell=1}^{L}\left|\left\langle R^{\ell-1} d, g_{\gamma_{\ell}}\right\rangle\right|^{2}+\left\|R^{L} d\right\|^{2}$, which proves that the residue norm is decreasing. At this stage, the signal $d$ is modeled as a finite linear combination of $L$ atoms with an error $R^{L} d$. However, this model can be improved using the same dictionary, since $R^{L} d$ is generally not orthogonal to $\mathbf{V}_{L}$, the linear span of $\left\{g_{\gamma_{\ell}}\right\}_{1 \leq \ell \leq L}$ : it is only orthogonal to the last selected atom $g_{\gamma_{L}}$. The OMP algorithm corrects this shortcoming by computing orthogonal projections at each iteration. As in MP, at iteration $\ell$ the algorithm selects an atom $g_{\gamma \ell}$ solving (1), builds an orthogonal basis of $\mathbf{V}_{L}$ using the Gram-Schmidt orthogonalization, and uses it to compute the orthogonal projection of $d$ on $\mathbf{V}_{L}$. With OMP, the residue norm is also proven to decrease monotonically.

Greedy algorithms have already been applied to seismic data for several different purposes, such as filtering [47], linear 


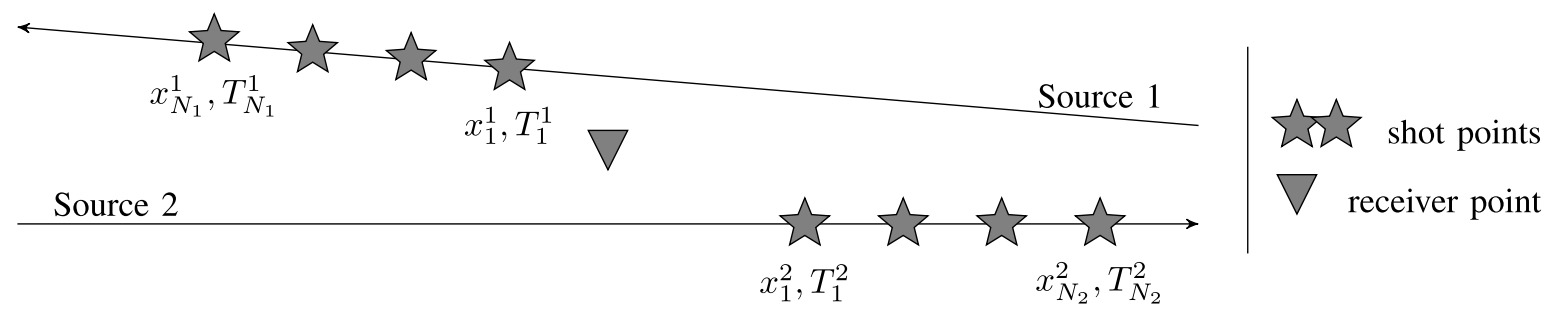

Fig. 1. Ocean Bottom Nodes (OBN) acquisition scheme for two seismic sources, where $T_{n}^{i}$ and $x_{n}^{i}$ denote the time and the coordinate of the $n$th shot of source $i$ on the axis of its shooting line.

noise suppression [48], [49], seismic data interpolation and regularization [50], [51], seismic data compression and sparse storage [52], [53], or reflectivity inversion [54], but few, if any, contributions have considered their application to the problem of separating signals from different sources.

\section{Modeling Sensor Signals in Simultaneous Sources SEISMic SuRveY}

We introduce our model of simultaneous-source seismic surveying and highlight the assumptions that justify our datadriven model for simple geometries of the Earth's subsurface.

\section{A. Earth's Transfer Function}

We consider an ocean bottom seismic acquisition with $K \geq 1$ sources $\left\{S_{k}\right\}_{1 \leq k \leq K}$ and a single sensor $D$ located at fixed positions in the Earth's space-time referential. We denote the $k$ th source excitation by $s_{k}(t)$, and the measured signal by $d(t)$, both time-dependent. The Earth acts as a filter (i.e., a linear, time-shift invariant, and continuous system) for the emitted signals $s_{k}(t)$, which enables us to represent the recorded signal as a convolution product $d(t)=\sum_{k=1}^{K}\left(r_{k}\right.$ 夫 $\left.s_{k}\right)(t)$, with the Earth's response coefficients $r_{k}$ depending on the positions of all the sources and the detector. Each source $S_{k}$ makes $N_{k}$ shots at times $\left\{T_{n}^{k}\right\}_{n \in \llbracket 1 ; N_{k} \rrbracket}$ and in the corresponding positions $\left\{\mathbf{x}_{n}^{k}\right\}_{n \in \llbracket 1 ; N_{k} \rrbracket}$. We also make the following hypothesis.

Hypothesis 1: Source $S_{k}$ emits the same short excitation $s_{k}$ for each of its shots.

Consequently, the signal $d(t)$ is given by

$$
d\left(\mathbf{x}_{D}, t\right)=\sum_{k=1}^{K} \sum_{n=1}^{N_{k}}\left(r_{k}\left(\mathbf{x}_{n}^{k}, \mathbf{x}_{D}\right) \star s_{k}\right)\left(t-T_{n}^{k}\right)+b(t)
$$

where $b(t)$ is the additive noise capturing the unavoidable imperfections of real seismic acquisitions. Note that $r_{k}\left(\mathbf{x}_{n}^{k}, \mathbf{x}_{D}\right)$ from (2) does not correspond to the true Earth reflectivity between $\mathbf{x}_{n}^{k}$ and $\mathbf{x}_{D}$ (the detector position) but acts as a transfer function between the source and receiver locations that accumulates Earth's entire response. Since the position of the detector is constant, we will write $d(t)$ instead of $d\left(\mathbf{x}_{D}, t\right)$.

\section{B. Simultaneous Sources for Classical Seismic Survey Design}

1) Experimental Conditions for Simultaneous-Source Surveys: We assume that each receiver continuously records all the seismic signals produced during the acquisition, which requires that all the survey equipment be kept synchronized. Time ranges from 0 to $T_{\text {glob }}$, the global acquisition time. We make the following hypothesis.

Hypothesis 2: The sources fire along straight lines, which may differ for different sources.

As illustrated in Fig. 1, the moveout of each seismic event depends on the source location along its shooting line. After sampling with period $\Delta_{t}$, the recorded data have the shape of a column matrix $d(k)=d\left(k \Delta_{t}\right)$. This type of recording is specific to simultaneous sources surveys. We further make two realistic hypotheses to simplify our analysis.

Hypothesis 3: Each source makes pauses between consecutive shots, during which its emitted signal is null.

Hypothesis 4: Shooting times of different sources are asynchronous and shooting intervals of each source are random.

The benefit of Hypotheses 3 and 4 is illustrated in Fig. 2, in which we align the data according to the shooting times of different sources. Each shot of the same source can be distinguished from the others following the time axis and the shots of different sources can be separated using a spatial coherence criterion-as detailed in Section II-C-which consists in a straight or slightly curved feature in the representation space $(t, x)$ of the data.

2) $(t, x)$ Representation Spaces of the Data: We define a linear operator, called pseudo-deblending, to align the sensor signal by the source $i$ to form the traces (see Fig. 3). For $d(t)$ from (2), it is written as $\mathcal{A}_{i}: L^{2}(\mathbb{R}) \rightarrow L^{2}\left(\left[0, \max _{n}\left(T_{n+1}^{i}-\right.\right.\right.$ $\left.\left.\left.T_{n}^{i}\right)\right] \times\left[x_{\min }^{i}, x_{\max }^{i}\right]\right), d(t) \mapsto \mathbf{D}_{i}\left(t^{\prime}, x\right)=d\left(t^{\prime}+T_{n}^{i}\right)$, if $x=$ $x_{n}^{i}-x_{0}^{i}$ and $t^{\prime} \in\left[0 ; T_{n+1}^{i}-T_{n}^{i}\left[; \mathbf{D}_{i}\left(t^{\prime}, x\right)=0\right.\right.$, otherwise. Pseudo-deblending creates as many data representation spaces $(t, x)$ as there are sources. To simplify, the $(t, x)$ representation space is called in the following a $(t, x)$ trace domain.

In conventional single source seismic, the operation $\mathcal{A}_{i}$ is done implicitly: the data are cut into traces according to the shooting times $T_{n}^{i}$, which do not play any further role in the processing. In contrast, for simultaneous-source data processing, it is crucial to preserve the shooting times, as they contain critical information to separate the signals coming from different sources.

We introduce these notions to clarify the concept of a seismic event, used in our data-driven model and related to the notion of traveltime curve, the graph of the time that a seismic wave spends to travel from the shot point to the receiver point. Note that the knowledge of the firing times and positions of each source makes it easy to switch from a 1-D representation to any 2-D trace domain representation of 

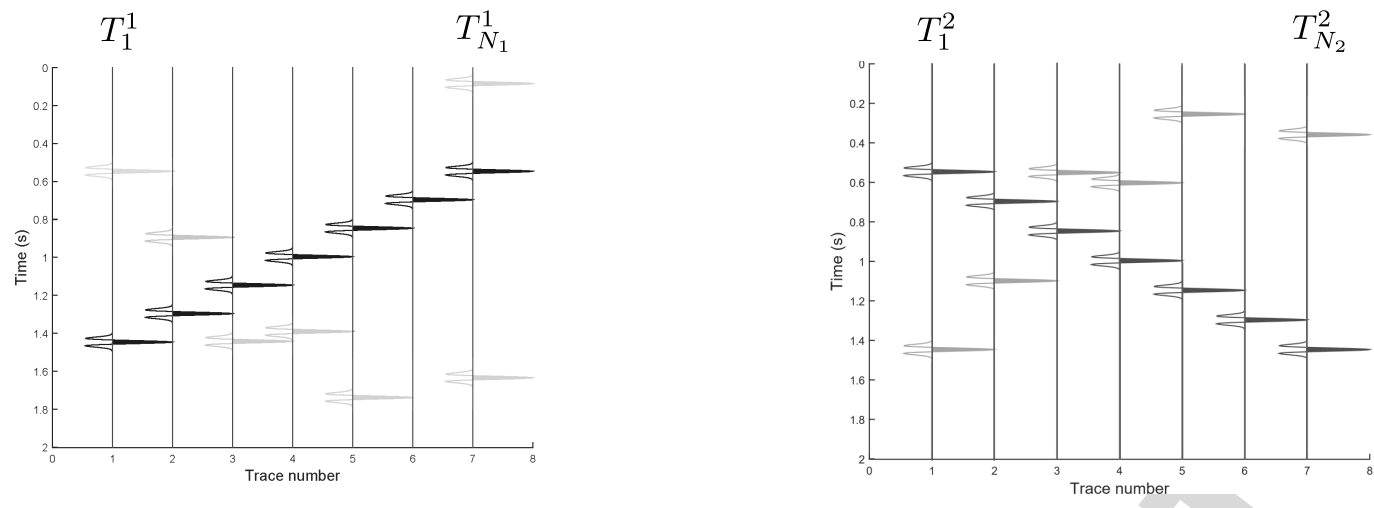

Fig. 2. Separation by random shot-times. Data aligned according to the (Left) first and (Right) second source shot-times. Dark (resp. light) wiggles correspond to the aligned (resp. nonaligned) source.

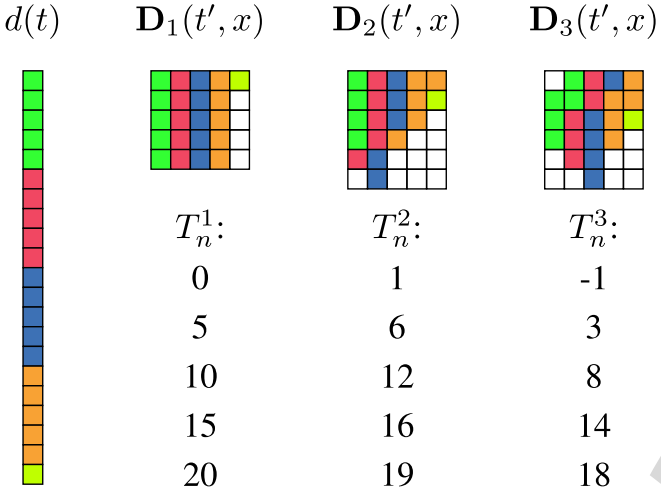

Fig. 3. Example of $(t, x)$ representations of a continuous signal cut into traces with regular shooting times $T_{n}^{1}$ for the first source and irregular ones $T_{n}^{2}$ and $T_{n}^{3}$ for the second and the third sources, respectively. Zero padding (the white squares correspond to zeros) is applied in order to keep the matrices rectangular. the signals and vice versa. Therefore, with a slight abuse of language, we use the same terminology for events and patterns in 1-D and 2-D representations, even though such events or patterns are only clearly visible in 2-D representations.

Hypothesis 5: Traveltime curves of coherent seismic waves (e.g., direct waves, surface waves, and reflected waves) are identifiable in one (and only one) seismic traces domain.

Traveltime curves are usually close to straight lines, parabolas, or hyperbolas in synthetic and real seismic data [55]. This observation and Hypothesis 5 imply the possibility of decomposing $d(t)$ into a sum of a finite number of coherent features that have a reasonably simple mathematical representation, as we shall see in Section II-C.

\section{Data-Driven Seismic Event Model}

We now introduce our parametric model of a seismic event that may either carry information about the Earth's subsurface geometry or correspond to a direct arrival. This model, which includes a curvature parameter, a magnitude attenuation factor depending on the source positions and the wavelet's decomposition into a sum of simple signals, and its implementation are the main contributions of this article.

1) Decomposition Into a Sum of Seismic Events: Actual seismic data usually have a significant size: one gather can contain hundreds of traces acquired with maximal offsets of $6 \mathrm{~km}$ or more. In complex geological environments with lateral velocity and density variability, it is difficult to establish a data-driven seismic model that would directly apply to the whole gather. Therefore, we choose to restrict our area of search to $N$ seismic traces in the $(t, x)$ domain, with $N$ typically between 10 and 30 depending on the data complexity. This allows us to make the following reasonable hypothesis.

Hypothesis 6: The wavelet $w(t)$ found in the data does not vary significantly from one seismic trace to another within some constrained spatial window of $N$ seismic traces.

When dealing with multiple sources recorded by the same receiver, which results in multiple $(t, x)$ trace domains to consider, one must adopt a consistent decomposition strategy. There may be several relevant ones, such as fully explaining all coherent features in the first source before passing on to the second one. If we were to follow this strategy, we would be able to cut our data into traces once, using the $\mathcal{A}_{i}$ operator for each source $i$, and continue with the 2-D $(t, x)$ trace domain representation common for a geophysicist. This approach has the following disadvantage: the algorithm aims at retrieving the low-amplitude signal hidden by the high-amplitude blending noise originating from the other sources. We propose to simultaneously work in all the $(t, x)$ trace domains in order to first identify and subtract the globally most energetic features and then continue with less energetic ones. The less energetic features are initially hidden under the crosstalk but are revealed by the first iterations of the algorithm. This is the main reason why we stick to the 1-D representation of the data. The decomposition is therefore simultaneously performed in all the sources $(t, x)$ trace domains, in which we look for particular identifiable features that we call seismic events. To do so, we first have to find in the column matrix $d(k \Delta t)$ the $N$-traces part of the signal corresponding to each of the sources. We then represent the data $d(t)$ as a finite sum of seismic events $h_{\ell} \star w_{\ell}(t)$

$$
d(t)=\sum_{\ell=1}^{L} h_{\ell} \star w_{\ell}(t)+R^{L} d(t) .
$$

Our model consists of two parts: $h(t)$, called traveltime curve (we call it curve because of the trace representation $(t, x)$ of 1-D signals), contains all the parameters related to the wave 
propagation time (medium characteristics), distance between the sources and the receiver, the sources firing times and the linear amplitude variation from one trace to another; $w(t)$, called signature or wavelet, is associated with the excitations emitted by the sources and distorted by propagation and reflection. Note that even if (2) and (3) are similar, there is a significant difference between the reflectivity $r_{i}\left(\mathbf{x}_{n}^{i}, \mathbf{x}_{D}\right)$, which is Earth's transfer function between the locations of the detector and of the $n$th shot of source $i$, and the traveltime curve $h(t)$, which indicates the position of a seismic event in the traces domain and is driven by the data. Moreover, the residue $R^{L} d(t)$ generally differs from the noise $b(t)$. In the case of two sources, we rewrite (3) as

$d(t)=\sum_{\ell=1}^{K_{1}} h_{\ell}^{(1)} \star w_{\ell}^{(1)}(t)+\sum_{\ell=1}^{K_{2}} h_{\ell}^{(2)} \star w_{\ell}^{(2)}(t)+R^{L} d(t)$

with $K_{1}+K_{2}=L$ and where the first (resp. second) sum corresponds to the seismic events identifiable in the $(t, x)$ traces domain of the first (resp. second) source. Thus, a perfect deblending would consist in reducing the residue $R^{L} d(t)$ to the ambient noise, as in this case, each sum would correspond to the isolated signal of the corresponding source. Before developing this point in Section III, we clarify the concepts of traveltime curve and wavelet in Sections II-C2 and II-C3.

2) Traveltime Curve Model: If we omit the amplitude variation, a traveltime curve is a graph of arrival time depending on the coordinates of the detector and the source shots. One can prove [55] that, for a simple case of a single horizontal reflector with a constant velocity above it, the traveltime curve is a hyperbola. Furthermore, with reasonable accuracy, one can model the arrival time function of a coherent seismic wave as a straight or slightly curved line in the $(t, x)$ trace domain within some lateral processing window (the closer the shot is to the receiver, the more curvature is observed). This assumption holds if the acoustic and elastic properties of the subsurface do not abruptly change in the horizontal direction within the chosen lateral processing window. The "pure" traveltime part $\tilde{h}(t)$ of the seismic event takes the form

$\tilde{h}^{(i)}\left(x_{n}^{i}, t\right)=\delta\left(t-\tau-p\left(x_{n}^{i}-x_{0}^{i}\right)-q\left(\frac{x_{n}^{i}-x_{0}^{i}}{x_{\max }^{i}-x_{\min }^{i}}\right)^{2}\right)$

or, for the convenience of our computation, in 1-D

$\tilde{h}^{(i)}(t)=\sum_{n=1}^{N} \delta\left(t-\tau-p\left(x_{n}^{i}-x_{0}^{i}\right)-q\left(\frac{x_{n}^{i}-x_{0}^{i}}{x_{\max }^{i}-x_{\min }^{i}}\right)^{2}-T_{n}^{i}\right)$.

Equations (5) and (6) are equivalent, but we stick to the 1-D representation to highlight the specific nature of the simultaneous-source data. Note that we omit the index $\ell$ present in (3) to alleviate notation. Here, $i$ is the index of the source associated with the event; $N$ the number of shots taken into account to construct the event; $x_{0}^{i}, x_{\min }^{i}$ and $x_{\max }^{i}$ are the reference coordinates of the $i$ th source; $\delta(t)$ is the Dirac distribution; $\tau, p$, and $q$ are the parameters that define the seismic event: the reference time, the slope and the curvature.
Finally, to obtain the full traveltime curve, we add a linear amplitude variation parameter $\alpha$ to this representation and obtain

$$
\begin{aligned}
h^{(i)}(t)= & \sum_{n=1}^{N}\left[1+\alpha\left(x_{n}^{i}-x_{0}^{i}\right)\right] \\
& \times \delta\left(t-\tau-p\left(x_{n}^{i}-x_{0}^{i}\right)-q\left(\frac{x_{n}^{i}-x_{0}^{i}}{x_{\max }^{i}-x_{\min }^{i}}\right)^{2}-T_{n}^{i}\right) .
\end{aligned}
$$

Note that the attenuation factor $1+\alpha\left(x_{n}^{i}-x_{0}^{i}\right)$ cannot vanish when $x_{n}^{i}=x_{0}^{i}$ in (7). We shall see in the following (Section III-B and criterion (13)) how one can address such a case. Strictly speaking, (7) defines an amplitude-variationpreserving traveltime curve, but for brevity we use the term traveltime curve in the following. It is worth noting that different sources illuminating the same area in the subsurface, e.g., an interface between two geological layers approximately at the same location, correspond to a single physical (geological) event; however, with our model (4), we obtain at least one separate seismic event per source. Moreover, even though our seismic event atoms correspond to simple cases of physical events, their linear combinations allow us to model complex physical situations (see Section IV).

3) Wavelet Model: Wavelet estimation has been a longstanding issue in seismic prospecting and different methods have been suggested in the literature. We focus on methods based on coherence; in other words, from Hypothesis 6, we assume that the wavelet does not abruptly change from trace to trace in a seismic event. This is intuitively justified by Hypothesis 1, and the fact that Earth's response to excitations varies slowly with respect to the source displacement. Nevertheless, we take into account the eventual presence of low and high energy noise that may perturb the wavelet originating from a single source by averaging the wavelet encountered in neighboring traces after getting rid of eventual outliers. Since propagation and reflection distort the source signals, the wavelet encountered in seismic gathers differs from the signal emitted by the source, and we suppose that the wavelet differs from one seismic event to another, even if it originates from the same seismic source.

As already mentioned, a single physical event may be captured by a sum of several seismic events, so we look for a new seismic event within a limited time interval that we denote $\left[-M \Delta_{t}, M \Delta_{t}\right]$ and call "corridor" ( $M$ is an integer meta-parameter). This corridor is defined along a traveltime curve of the form (7), which is assumed known for now. It must be large enough not to change the wavelet spectrum. The fact that the traveltime curve $h^{(i)}$ is not perfectly known is addressed in Sections III-B and III-D. After getting a first estimation $\hat{w}^{(i)}$ of the wavelet $w^{(i)}$, we refine the estimation thanks to optimization stages described in Section III-C2. To reduce the dimension of the optimization problem, we choose to decompose the estimated wavelet into a linear combination of a small number of wavelet atoms. We also choose wavelet atoms that can be represented analytically, e.g., Ricker and Ormsby wavelets, which are elementary wavelets widely used in seismic exploration and for which 


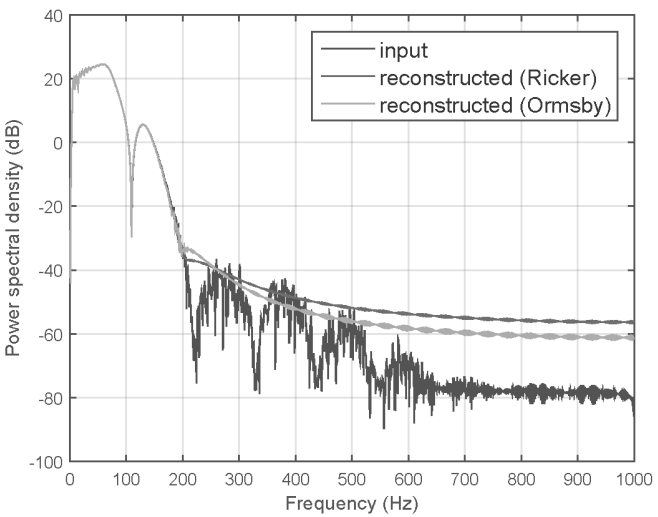

Fig. 4. Power spectrum of the signal of length $500 \mathrm{~ms}$ in black, reconstructed using 178 Ricker (in dark gray), or 212 Ormsby (in light gray), wavelets.

we can explicitly compute temporal derivatives. This allows us to reduce the computational complexity of our algorithm optimization stages.

We choose to decompose the estimated wavelet into a linear combination of a small number of wavelet atoms using the OMP algorithm, which requires the identification of an adapted dictionary. We shall see in Section III-C1, how we construct a finite number $S$ (of several units) of classical wavelet shapes from a preliminary spectral analysis of the data. The index $s$ denotes the shape of the wavelet $w_{s}(t)$, and the dictionary consists of atoms (before normalization) $\left\{w_{s}(t-\tau): 1 \leq\right.$ $s \leq S, \tau \in[0, T]\}$ where $T>0$ is a meta-parameter. Thus, we obtain the following parametric wavelet estimation:

$$
\begin{aligned}
& \hat{w}^{(i)}(t)=\sum_{k=1}^{K} a_{k} w_{s_{k}}\left(t-\tau_{k}\right)+R^{K} \hat{w}^{(i)}(t) \quad \text { and } \\
& w^{(i)}(t)=\sum_{k=1}^{K} a_{k} w_{s_{k}}\left(t-\tau_{k}\right) .
\end{aligned}
$$

Fig. 4 shows the power spectrum of a modeled marine seismic source signature. We observe that the parametric model of the form (8) is accurate enough in the useful part of the spectrum both with Ricker ( $K=178$ for this example) and Ormsby $(K=212)$ wavelets. Note that these numbers are significantly larger than those used in our deblending algorithm because here the whole length of the source signal is taken into account $(0.5 \mathrm{~s})$ with a very dense sampling $(0.5 \mathrm{~ms}$ of period). For subsequent simulations we use narrower corridors for wavelet estimation, typically $0.1 \mathrm{~s}$, with $\Delta_{t}=2 \mathrm{~ms}$.

\section{MATRioshKa OMP IMPLEMENTATION}

We now present the implementation of our algorithm. In Section III-A, we present the fundamentals of our method, which we call Matrioshka OMP and which relies on detailed parameter optimization. To obtain suitable parameter values, we use iterative optimizations, which require a sufficiently accurate prior knowledge of the parameters, i.e., satisfactory initial conditions. The initial condition computation is described in Section III-B. Sections III-C and III-D provide an overview of the different parts of the algorithm.

\section{A. Deblending Using Data-Driven Model and OMP}

An iterative method that performs a decomposition as in (4) automatically results in a partial deblending of the data. Moreover, if the first terms in this decomposition correspond to the seismic events having the most energy, then only the lowest energy cross-talks are left in the residue $R^{L} d(t)$, which can then be handled by classical seismic processing techniques as if no other sources had been firing simultaneously. Consequently, we look for a decomposition (4) in which the most energetic features of the deblended signal associated with the $i$ th source are found in the sum

$$
\sum_{\ell=1}^{K_{i}} h_{\ell}^{(i)} \star w_{\ell}^{(i)}
$$

and the most energetic cross-talks due to the other sources are captured in the sums

$$
\sum_{\ell=1}^{K_{j}} h_{\ell}^{(j)} \star w_{\ell}^{(j)} \quad \text { with } j \neq i
$$

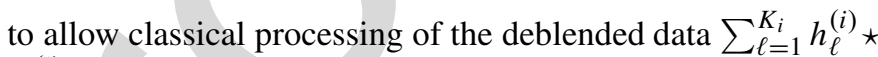
$w_{\ell}^{(i)}+R^{L} d(t)$. To successfully deblend with this approach, it is crucial that the sum (9) contains the most energetic features of the deblended signal associated with the source $i$ and not any coherent seismic events originating from a source $j \neq i$. Hypotheses 2, 3, and 4 justify the fact that we can expect to capture in the sum (9) seismic events originating from the source $i$ alone. Moreover, if the most energetic features are identified at the first iterations of the decomposition, then the most energetic cross-talks from other sources are captured in other sums (10), and thus, do not pollute the residue $R^{L} d(t)$ any more. Fortunately, this is exactly how OMP proceeds provided that we choose a well-adapted dictionary. Now, if the atoms are expressed, before normalization, as $\mathcal{G}_{\gamma}=h^{(i)} \star w^{(i)}$, and $h^{(i)}$ and $w^{(i)}$ given, respectively, by (7) and (8), then

$$
\begin{aligned}
& \mathcal{G}_{\gamma}(t) \\
& =\sum_{n=1}^{N}\left[1+\alpha\left(x_{n}^{i}-x_{0}^{i}\right)\right] \sum_{k=1}^{K} a_{k} \\
& \quad \times w_{s_{k}}\left(t-\tau-p\left(x_{n}^{i}-x_{0}^{i}\right)-q\left(\frac{x_{n}^{i}-x_{0}^{i}}{x_{\max }^{i}-x_{\min }^{i}}\right)^{2}-T_{n}^{i}-\tau_{k}\right)
\end{aligned}
$$

with $\gamma=\left\{i, \tau, p, q, \alpha, K,\left\{s_{k}, a_{k}, \tau_{k}\right\}_{1 \leq k \leq K}\right\}$ the complete set of parameters. Hence, we can construct a decomposition (4) that fulfills the aforementioned conditions required for deblending. Examples of such atoms, given in Fig. 5, show the ability of the algorithm to handle curvature and amplitude variation.

Note that, when $L$ tends to infinity, the residue $R^{L} d$ is not necessarily white noise or any other type of noise. Indeed, it corresponds to the last residue (part of the signal) not explained by the dictionary, i.e., orthogonal to the dictionary used for decomposition. A "good" decomposition, though, would leave the noise in the residue. 

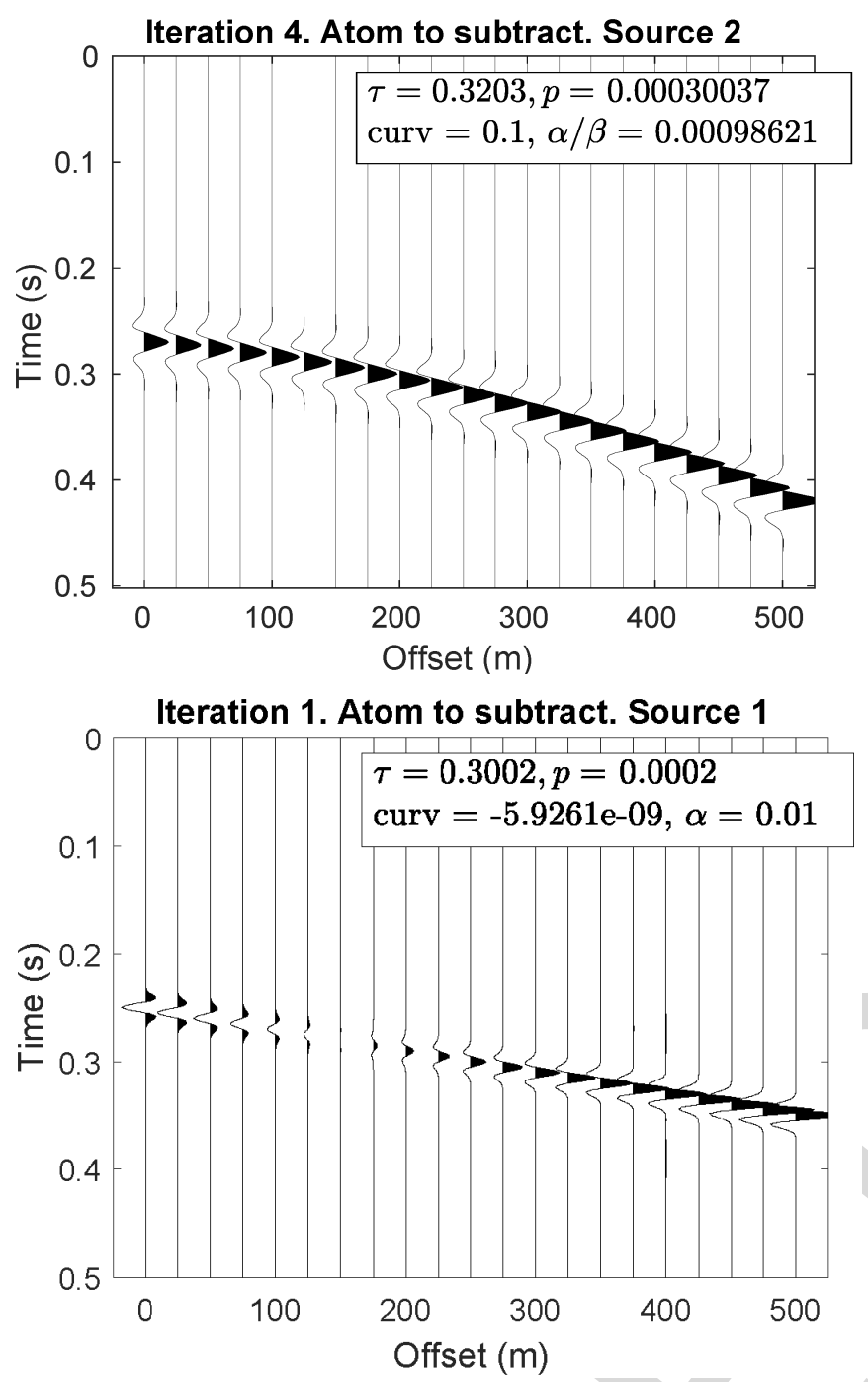

Fig. 5. Examples of atoms $\mathcal{G}_{\gamma}(t)$ (before normalization) of the seismic events dictionary.

To simplify the computation of vector norms $\left\|\mathcal{G}_{\gamma}\right\|$, we make the following hypothesis.

Hypothesis 7: For each source, the pauses between two consecutive shots are significantly longer than the emission time of each shot of the same source.

Note that Hypothesis 7 does not forbid crosstalk between consecutive shots of the same source, i.e., the delay between consecutive shots can be smaller than the listening time implying auto-pollution or self-simultaneous sourcing.

Now, the problem is to find an approximate solution of (1). For this, we must overcome two major difficulties: 1) the objective function to maximize is not concave and 2) the number of parameters describing an atom is too large for sampling the dictionary into a finite subset of atoms $\Gamma \subset \Omega$. To overcome the first difficulty, we use iterative optimization algorithms that converge to a local maximum whose position depends on the initial conditions. It is therefore crucial to accurately choose the initial conditions. To overcome the second difficulty, we gradually build atoms of the dictionary close to the desired maximum.

\section{B. Initial Conditions of the OMP Optimization Step}

In this section, we present our approach to find the initial conditions of the iterative algorithm. We construct an atom $\mathcal{G}_{\gamma}$ (before normalization) given by (11) in several steps. We start by building the traveltime curve $h^{(i)}$ given in (7), first looking for parameters $i, \tau, p, q$ that maximize the objective function

$$
\begin{aligned}
& C(i, \tau, p, q) \\
& \quad=\left|\sum_{n=1}^{N} R^{\ell-1} d\left(\tau+p\left(x_{n}^{i}-x_{0}^{i}\right)+q\left(\frac{x_{n}^{i}-x_{0}^{i}}{x_{\max }^{i}-x_{\min }^{i}}\right)^{2}+T_{n}^{i}\right)\right| .
\end{aligned}
$$

In other words, noting that $C(i, \tau, p, q)=\left|\tilde{h}^{(i)} \star \widetilde{R^{\ell-1}} d(0)\right|$, for $\tilde{h}^{(i)}$ introduced in (6), and $R^{\ell-1} d(t)=R^{\ell-1} d(-t)$, we are looking for a traveltime curve $\tilde{h}^{(i)}$ that maximizes the magnitude of its correlation at time $t=0$ with the residue $R^{\ell-1} d(t)$ at the $\ell$ th OMP iteration. Here too, the objective function is not concave, and the parameters $i, \tau, p, q$ that maximize (12) are found using an iterative optimization algorithm starting from suitable initial conditions and converging to a local maximum. To do so, we introduce the following hypothesis.

Hypothesis 8: To maximize the objective function in (12), good initial conditions are $q=0$ and the values of $i, \tau, p$ that maximize the slant stack magnitude of the residue $R^{\ell-1} d$.

Various successful applications of slant stack (or Linear Radon Transform) to seismic data processing justify this hypothesis, for example, coherent noise suppression, such as multiples and direct arrivals removal [56]; plane-wave decomposition for velocity picking [57]. In our case, if one can pick the absolute maximum in the $(\tau, p)$ domain, this maximum identifies a real seismic event with nearly the most energy. Once we have identified a traveltime curve $\tilde{h}^{(i)}$ that maximizes its correlation with the residue, we compute the coefficients $\alpha^{\prime}$ and $\beta^{\prime}$ of the linear regression between the term $R^{\ell-1} d\left(\tau+p\left(x_{n}^{i}-x_{0}^{i}\right)+\cdots\right)$ appearing in (12) and $x_{n}^{i}-x_{0}^{i}$ for $1 \leq n \leq N$ that minimize

$$
\begin{aligned}
& C \sum_{n=1}^{C\left(\alpha^{\prime}, \beta^{\prime}\right)}\left[R^{\ell-1} d\left(\tau+p\left(x_{n}^{i}-x_{0}^{i}\right)+q\left(\frac{x_{n}^{i}-x_{0}^{i}}{x_{\max }^{i}-x_{\min }^{i}}\right)^{2}+T_{n}^{i}\right)\right. \\
& \left.\quad-\left[\beta^{\prime}+\alpha^{\prime}\left(x_{n}^{i}-x_{0}^{i}\right)\right]\right]^{2}
\end{aligned}
$$

in order to obtain a first estimation of the complete traveltime curve $h^{(i)}$ given in (7). In addition, when $\left|\beta^{\prime}\right|>\varepsilon$ (in our implementation we took $\varepsilon=10^{-7}$ ), we set $\alpha=\alpha^{\prime} / \beta^{\prime}$.

We observed that criterion (12) does not give the best initial conditions to the OMP optimization when the factor $\beta^{\prime}+\alpha^{\prime}\left(x_{n}^{i}-x_{0}^{i}\right)$ changes its sign between the extreme values of $x_{n}^{i}$ and we shall see in Section III-E how to modify (12) to obtain better initial conditions.

Next, we define a "corridor" in the representation space $(t, x)$ associated with the $i$ th source. This corridor has a width of $(2 M+1) \Delta_{t}$, it is centered around the maximal values of $\tilde{h}^{(i)}$ and passes through the $N$ considered traces. We thus obtain a 
nonparametric estimation $\hat{w}^{(i)}$ of the wavelet $w^{(i)}$, associated with the atom $\mathcal{G}_{\gamma}$ introduced in (11). The estimation is locally made from the current residue, within the corridor and after making the following hypothesis.

Hypothesis 9: A wavelet estimation can be statistically derived from the $N$ traces by stacking along curves parallel to the traveltime curve maxima weighted by attenuation factors.

We then apply a Tukey window to this nonparametric wavelet estimation to avoid discontinuities at the corridor edges. Finally, we obtain a parametric estimation $w^{(i)}$ of the wavelet having the form (8) by applying the OMP algorithm to the windowed wavelet estimation, and we compute the nonnormalized atom $\mathcal{G}_{\gamma}$, mentioned at the beginning of this section, as $h^{(i)} \star w^{(i)}$.

We can summarize the computation of the initial conditions $\mathcal{G}_{\gamma}$ into the following stages. ${ }^{2}$

1) Find the values $i, \hat{\tau}$, and $\hat{p}$ that maximize the slant stack magnitude of the residue $R^{\ell-1} d$.

2) From the initial conditions obtained at the previous stage and $q=0$, find a traveltime curve $\tilde{h}^{(i)}$ maximizing its correlation magnitude with $R^{\ell-1} d$ at time $t=0$, within the $N$ traces.

3) Find the coefficients $\alpha^{\prime}$ and $\beta^{\prime}$ of the regression (13) to obtain $\alpha=\alpha^{\prime} / \beta^{\prime}$ and the amplitude-variation-preserving traveltime curve $h^{(i)}(t)$, with the attenuation factor $1+\alpha\left(x_{n}^{i}-x_{0}^{i}\right)$.

4) In the $(t, x)$ trace domain associated with source $i$, identify a $(2 M+1) \Delta_{t}$-seconds high corridor centered around the traveltime curve maxima found at the previous stage; then make a nonparametric wavelet estimation using a weighted stacking by reverse attenuation factors along the curves parallel to the $\tilde{h}^{(i)}$ maxima within the corridor.

5) Window the nonparametric wavelet estimation obtained at the previous stage and apply OMP to get a parametric estimation $w^{(i)}$ given by (8).

6) Find the initial conditions atom which, before normalization, equals to $\mathcal{G}_{\gamma}=h^{(i)} \star w^{(i)}$.

In this way, we propose to perform deblending by means of OMP, and we use the OMP algorithm twice. To distinguish them, we denote by outer OMP, the one which has a dictionary of atoms of the form (11) before normalization, and by inner OMP, the one performing the parametric wavelet estimation. In Section III-C, we present the inner OMP algorithm.

\section{Inner OMP Overview}

Before starting the iterations of Matrioshka OMP, we perform a spectral analysis of the data to determine the shapes of the wavelets to use in the inner OMP dictionary. For this, we compute the power spectrum of $d(t)$ and pick the frequency values at its maximum and 3 and $6 \mathrm{~dB}$ lower. This procedure provides a set of frequencies that we use to build the wavelet dictionary (for example, the five frequencies above give the dominant frequencies of the Ricker wavelets).

\footnotetext{
${ }^{2}$ We shall see in Section III-E that, when the factor $\beta^{\prime}+\alpha^{\prime}\left(x_{n}^{i}-x_{0}^{i}\right)$ changes its sign between the extreme values of $x_{n}^{i}$, the stages 2 and 3 can be iterated, modifying the criterion (12). For simplicity, we do not present this procedure here.
}

1) Wavelet Dictionary: We choose a finite number $S$ of classical wavelet shapes. The shape index $s(1 \leq s \leq S)$ corresponds to either a Ricker wavelet with a given dominant frequency or an Ormsby wavelet with a given set of cut-off frequencies. If we need Ricker wavelets of different dominant frequencies, we use as many Ricker shapes as needed and likewise for Ormsby wavelets. The predefined shapes can be extended to any other kind of wavelets.

The dictionary is composed of time-shifted unit-norm elementary wavelets of the predefined shapes. Since the estimated wavelet must be inside the abovementioned corridor, we limit the time shifts so that an atom is represented as $w_{\gamma}(t)=$ $\left(w_{s}\left(t-v \Delta_{\tau}^{\prime}-\tau^{\prime}\right)\right) /\left\|w_{s}(t)\right\|$, where $v \in \llbracket-\mu M, \mu M \rrbracket$ is an integer, $\left.\tau^{\prime} \in\right]\left(-\Delta_{\tau}^{\prime} / 2\right), \Delta_{\tau}^{\prime} / 2\left[\right.$ with $\Delta_{\tau}^{\prime}=\Delta_{t} / \mu$, and $\mu^{-1} \in \mathbb{N}$ divides $M$. Thus, the dictionary is $\mathcal{D}=\left\{w_{\gamma}\right\}_{\gamma \in \Omega}$ with $\Omega=\left\{\left(s, \nu, \tau^{\prime}\right): s \in \llbracket 1, S \rrbracket, \nu \in \llbracket-\mu M, \mu M \rrbracket\right.$ and $\left.\tau^{\prime} \in\right]\left(-\Delta_{\tau}^{\prime} / 2\right),\left(\Delta_{\tau}^{\prime} / 2\right)[\}$. We also use a discrete version of the dictionary, with vanishing $\tau^{\prime}:\left\{w_{\gamma}\right\}_{\gamma \in \Gamma}$ with $\Gamma=\{(s, v, 0) \in \Omega\}$.

2) Inner OMP: For simplicity, in this paragraph, we omit the superscript $(i)$ of an estimated wavelet $w^{(i)}$, and we consider wavelets as continuous-time signals.

The inner OMP is initialized with the windowed nonparametric estimation $R^{0} w(t)=\tilde{w}(t)$. Let $R^{k-1} w$ be the residue after $(k-1)$ iterations of the inner OMP. At iteration $k$, first we look for a solution $\hat{\gamma}_{k}=\left(s_{k}, v_{k}, 0\right)$ to $\left|\left\langle R^{k-1} w, w_{\hat{\gamma}_{k}}\right\rangle\right|=\max _{\gamma \in \Gamma}\left|\left\langle R^{k-1} w, w_{\gamma}\right\rangle\right|$, which gives initial conditions for the iterative optimization algorithm converging to a local maximum, approximate solution to $\left|\left\langle R^{k-1} w, w_{\gamma_{k}}\right\rangle\right|=\max _{\gamma \in \Omega}\left|\left\langle R^{k-1} w, w_{\gamma}\right\rangle\right|$. Thus, we obtain $w_{\gamma_{k}}$, the atom of the inner OMP chosen at the iteration $k$. In the following step, we update the coefficients of the orthogonal projection of $\tilde{w}$ on the vector subspace of the first $k$ atoms obtained via the inner OMP. After $K$ iterations, we obtain the decomposition $\tilde{w}(t)=\sum_{k=1}^{K} a_{k} w_{s_{k}}\left(t-v_{k} \Delta_{\tau}^{\prime}-\tau_{k}^{\prime}\right)+R^{K} \tilde{w}(t)$, which gives the parametric estimation of the stage 5 above: $w^{(i)}=\sum_{k=1}^{K} a_{k} w_{s_{k}}\left(t-v_{k} \Delta_{\tau}^{\prime}-\tau_{k}^{\prime}\right)$.

Section III-D presents a complete view of the deblending algorithm Matrioshka OMP.

\section{Matrioshka OMP Overview}

Matrioshka OMP [58] stands for two OMP algorithms embedded into one another. The algorithm is illustrated in Fig. 6, where the outer OMP consists of the whole algorithmic loop with the inner OMP embedded into it and highlighted in orange. We now describe each step individually.

After the spectral analysis of the data, the second stage of the processing is to split the continuously recorded signal $d(t)$ into temporal frames suitable for deblending. It is worth noting that the definition of windows width using a number of traces is no longer compatible with the data. Indeed, the number of traces (whole or parts) does not necessarily match for the different sources. To overcome this ambiguity, we chose to define window width in terms of time. When a window break occurs between shooting times of a source, we use the knowledge of the previous shooting time to exploit all the 


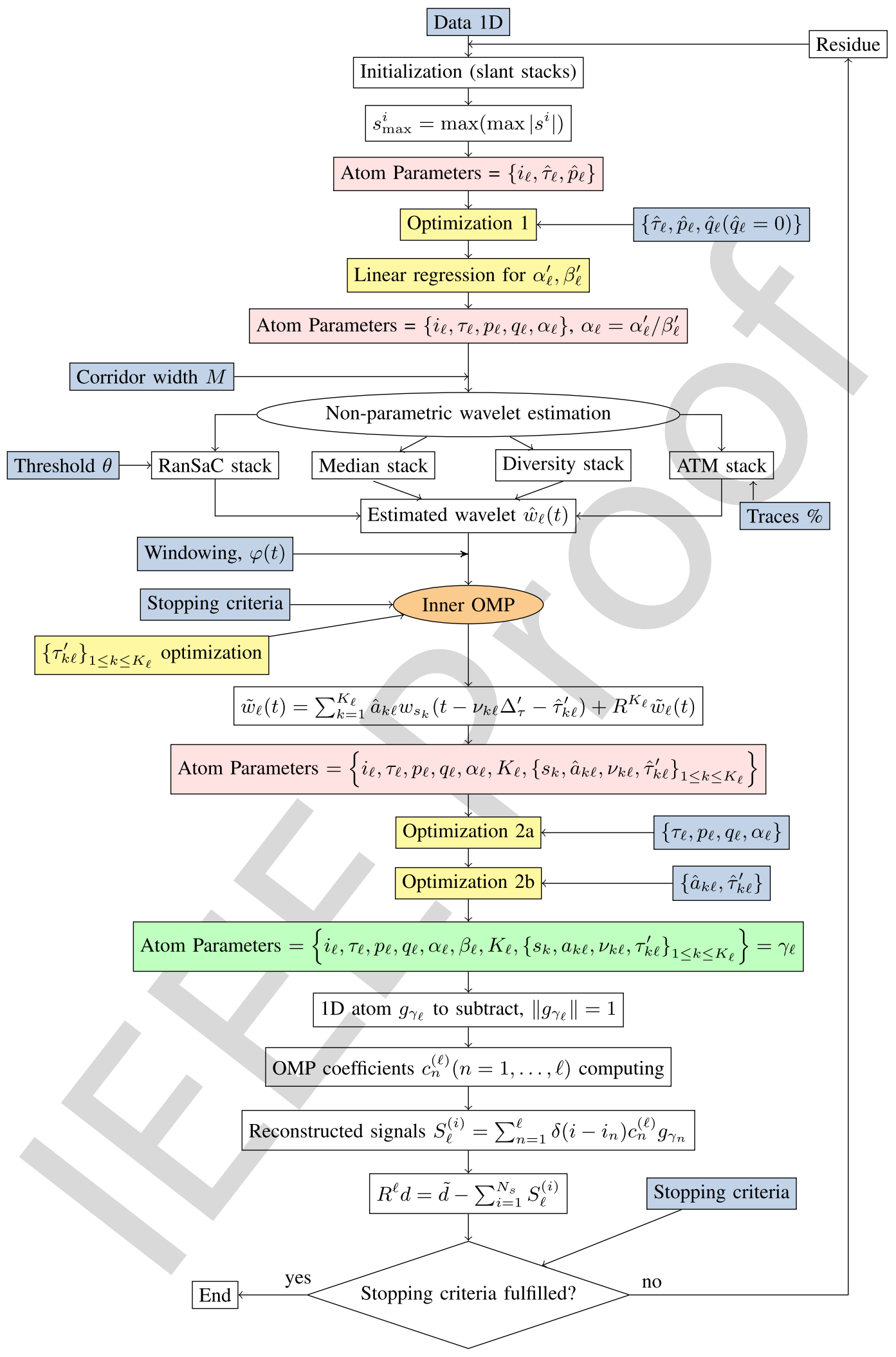

Fig. 6. Matrioshka OMP algorithm, with inputs, optimization steps, intermediate parameters

final parameters

information available in the data. Thus, the outer OMP is initialized from the input data $d(t)$ windowed by a rectangular time window strictly included in the interval $\left[0, T_{\text {glob }}\right]$ and corresponding to $N$ seismic traces for one source. We denote by $\tilde{d}(t)$ the windowed signal $d(t)$ and take it as the first 700 residue: $R^{0} d=\tilde{d}$.

Let $R^{\ell-1} d$ be the residue after $(\ell-1)$ iterations of ${ }_{702}$ the outer OMP. At the $\ell$ th iteration, we have seen in 703 
(a)
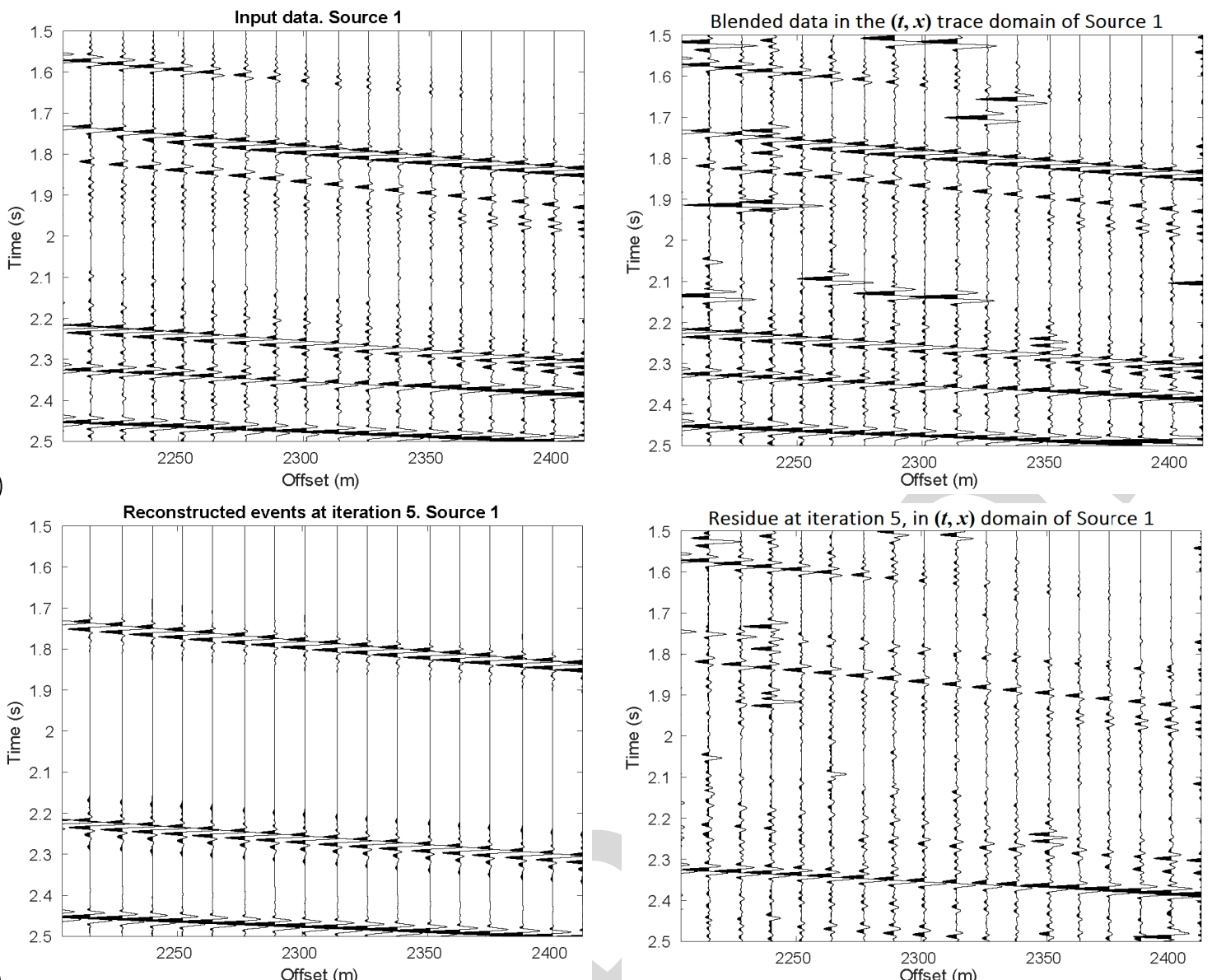

$(c)$
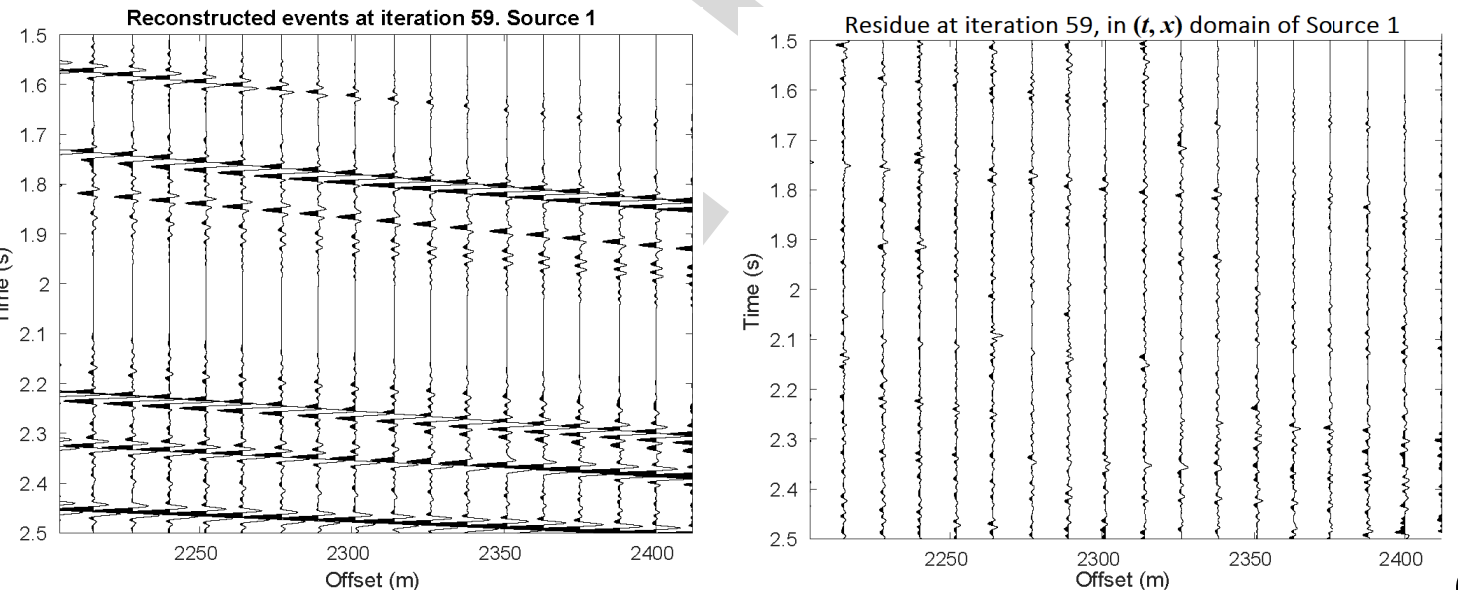

$(e)$

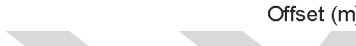

Fig. 7. (a) Input unblended data for the first source and (b) same data after blending. (c) Reconstruction results after $L=5$ iterations of the outer OMP: events attributed to the first source, i.e., $S_{\ell}^{(1)}$ of (14) with $\ell=L$ and (d) residue $R^{L} d$ of (4). (e) and (f) Idem after $L=59$ iterations. All the signals are represented in the $(t, x)$ trace domain of Source 1 . In our method, the deblended signal associated with Source 1 after $L$ iterations is the sum of signals appearing in the graphs (c) and (d) for $L=5$ and the sum of signals in the graphs (e) and (f) for $L=59$.

Sections III-B and III-C how to obtain the initial conditions (11) before normalization, which allow an iterative optimization algorithm to converge to a local maximum. Relationships allowing a fast computation of the norm of seismic events of the form (11) under Hypothesis 7 can be found in [59, Appendix C].

In order to separate travel-path-related parameters from the wavelet-defining ones, so that they do not intercompensate each other, we first optimize the $\tau, p, q$ and $\alpha$ parameters and then the $\left(a_{k}, \tau_{k}^{\prime}\right)_{1 \leq k \leq K}$ parameters. Note that the whole dictionary is never created or stored due to computational costs: a new element of the dictionary is estimated at each iteration. We obtain after these optimization stages the atom $g_{\gamma_{\ell}}$, approximate solution of (1), with $\gamma_{\ell}=\left(i_{\ell}, \tau_{\ell}, p_{\ell}, q_{\ell}, \alpha_{\ell}, K_{\ell}\right.$,

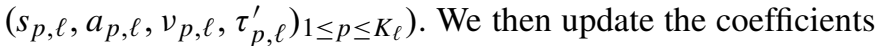
$\left(c_{p}^{(\ell)}\right)_{1 \leq p \leq \ell}$ of the orthogonal projection of $\tilde{d}$ on the linear 
(a)
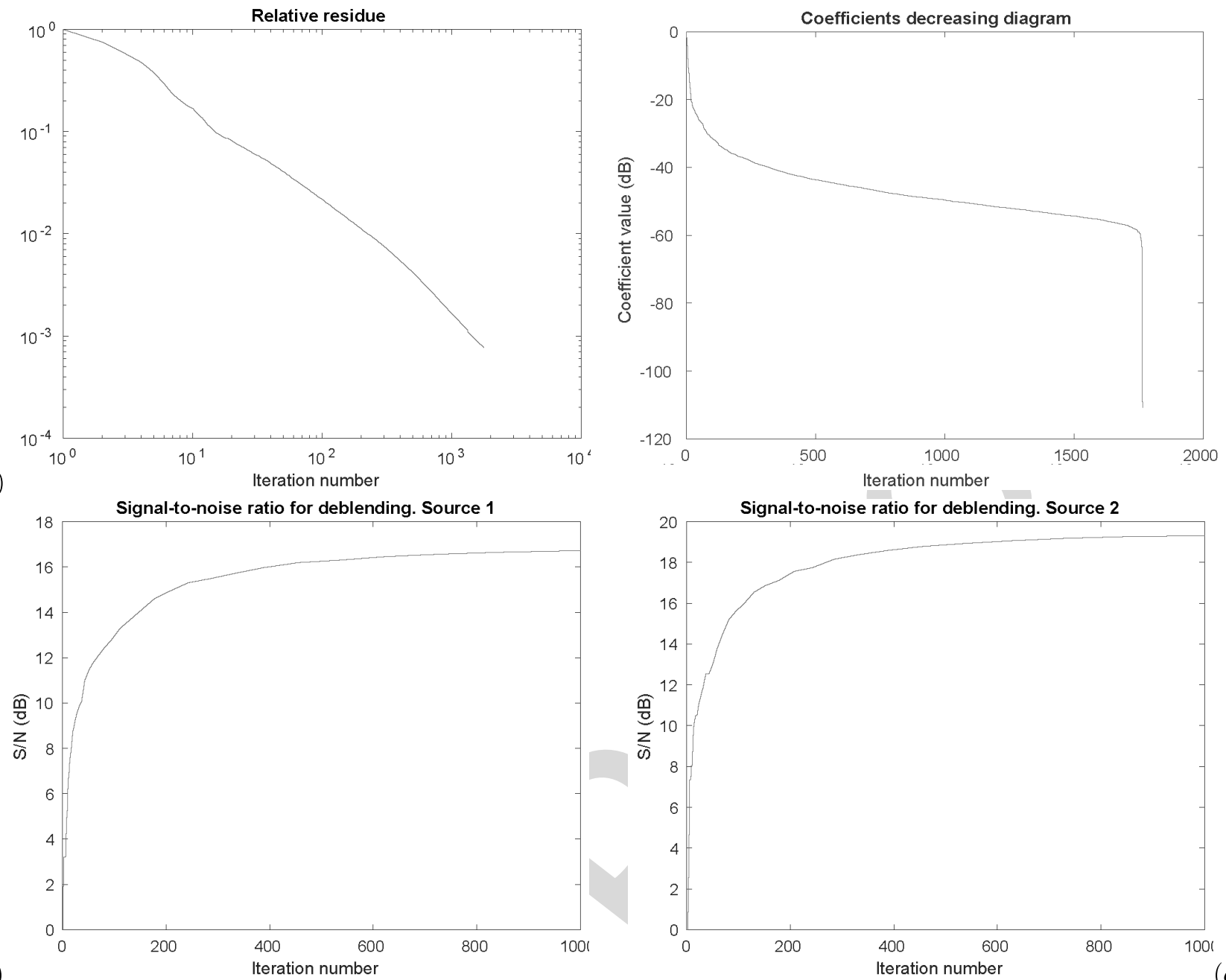

(c)

Iteration number

(d)

Fig. 8. (a) Results obtained on synthetic data with real seismic noise added. Residue energy decreasing in bilogarithmic scale. (b) Coefficients magnitude decreasing diagram. (c) and (d) Signal-to-noise ratio increasing with the outer OMP iterations for the two sources. The signal-to-noise ratio is computed as $\mathrm{S} / \mathrm{N}=10 \log _{10}\left(\left\|d_{s}\right\|^{2} /\left\|d_{s}-d_{d}\right\|^{2}\right)$, where $d_{s}$ denotes the initial single source data, and $d_{d}$ the deblended data for the same source.

subspace spanned by the first $\ell$ outer OMP atoms, and the weighted sums - called explained signals in the followingassuming we have $N_{s}$ sources:

$$
S_{\ell}^{(i)}(t)=\sum_{p=1}^{\ell} \delta_{i, i_{p}} c_{p}^{(\ell)} g_{\gamma_{p}}\left(\text { for source } i=1, \ldots, N_{s}\right)
$$

where $\delta_{i, j}$ is the Kronecker delta function. After $L$ iterations, $\tilde{d}(t)=\sum_{\ell=1}^{L} c_{\ell} g_{\gamma_{\ell}}(t)+R^{L} d(t)=\sum_{i=1}^{N_{s}} S_{L}^{(i)}(t)+R^{L} d(t)$, and the deblended signal associated with the $i$ th source is equal to $S_{L}^{(i)}(t)+R^{L} d(t)$. To reduce the computational complexity of the method, the optimization stages must be efficiently implemented. An asymptotic complexity analysis of the algorithm is given in [59]. After processing each temporal window, the deblended data are merged. To increase the deblending quality and avoid high-frequency residual noise, windows overlap. We end the section by presenting the initial condition computation when the maximum magnitude of the wavelet changes sign from one end of the seismic event to the other.

\section{E. Seismic Events With a Phase Rotation}

To find the initial conditions of $\mathcal{G}_{\gamma}$, the approach described in stages 2 and 3 of Section III-B works perfectly for seismic events which have the same polarity all along the processing window. However, it is common to encounter a "phase rotation" corresponding to events whose maxima have different signs on the left and on the right edge of the processing window (see Fig. 5). In this case, (12) no longer represents a good objective function to maximize because the algorithm tends to favour (to follow) amplitudes of the same sign. To solve this problem, we modified the criterion (12) to

$$
\begin{aligned}
& C(i, \tau, p, q) \\
& =\mid \sum_{n=1}^{N} R^{\ell-1} d\left(\tau+p\left(x_{n}^{i}-x_{0}^{i}\right)+q\left(\frac{x_{n}^{i}-x_{0}^{i}}{x_{\max }^{i}-x_{\min }^{i}}\right)^{2}+T_{n}^{i}\right) \\
& \quad \times \operatorname{sgn}\left[\beta^{\prime}+\alpha^{\prime}\left(x_{n}^{i}-x_{0}^{i}\right)\right] \mid .
\end{aligned}
$$



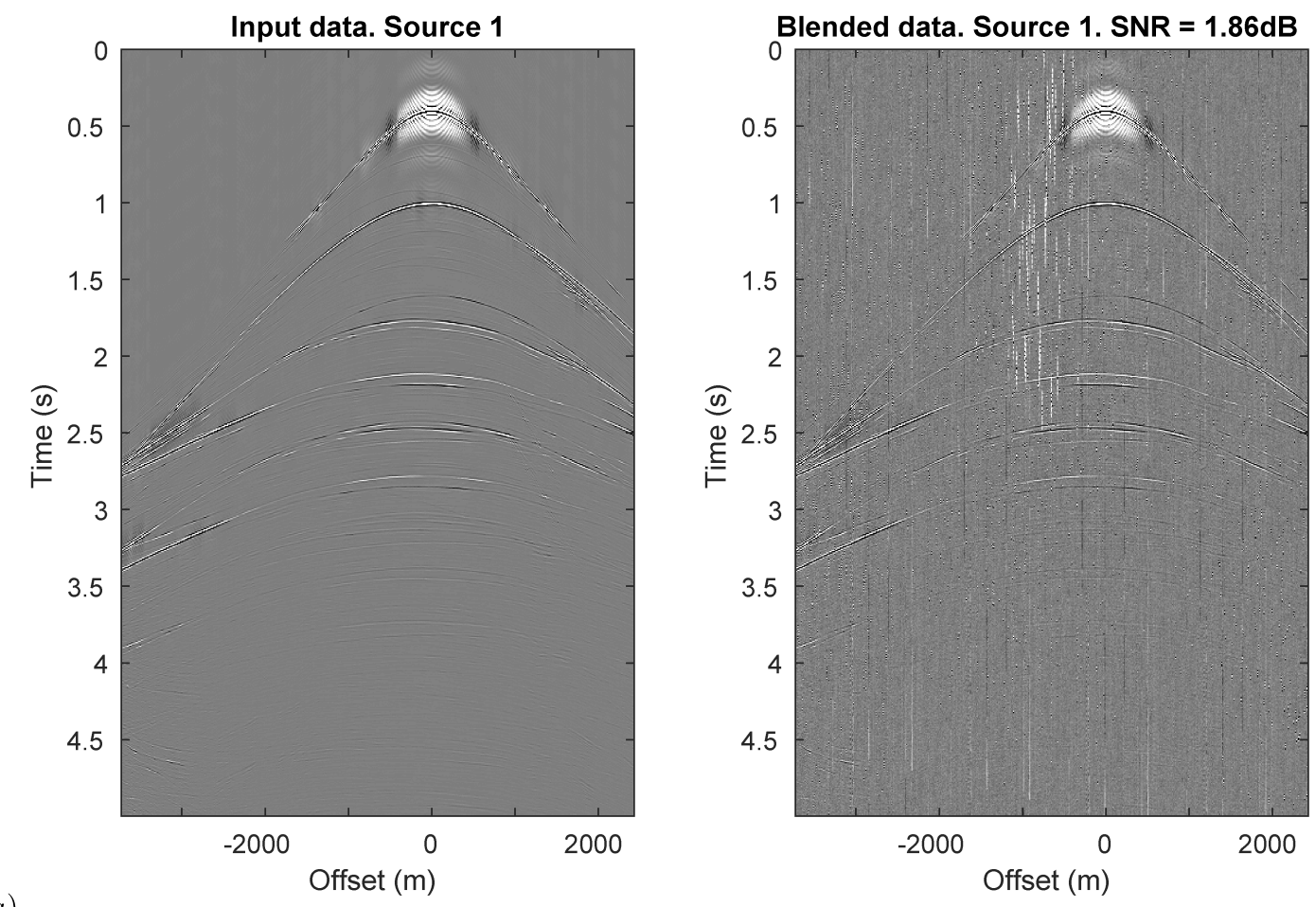

$(a)$
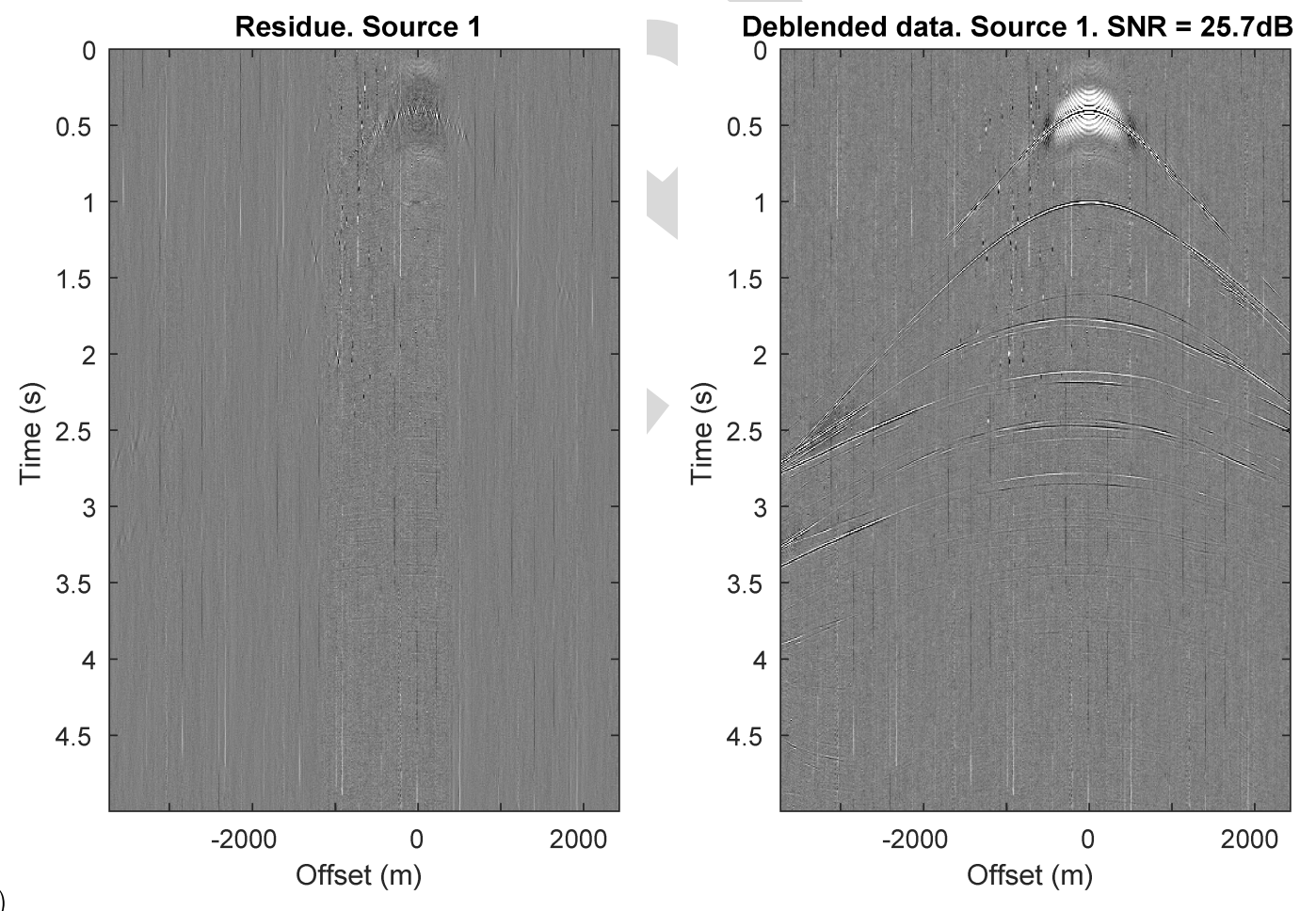

(b)

$(d)$

Fig. 9. (a) Full gather tests for the synthetic Marmousi data with added real seismic noise, the first source. Input nonblended signal and (b) same data after blending. (c) Residue after decomposition and (d) data after deblending (explained signal with the residue added).

and iterated twice the stages 2 and 3 ; this proved to be effective in our simulations.

\section{F. Stopping Criteria}

Due to the significant complexity of seismic data with respect to our dictionary, it is very difficult to define a single stopping criterion applicable everywhere. Moreover, the stopping criterion must be adapted to the downstream processing. For this reason, we propose setting multiple stopping criteria for each simulation to achieve more accurate results and, at the same time, avoid wasting machine time on unnecessary precision seeking. 


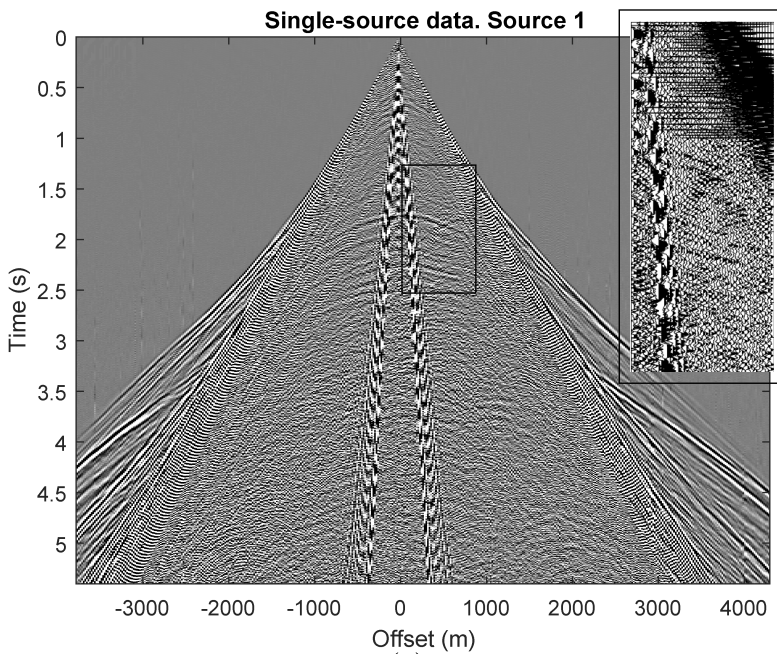

(a)

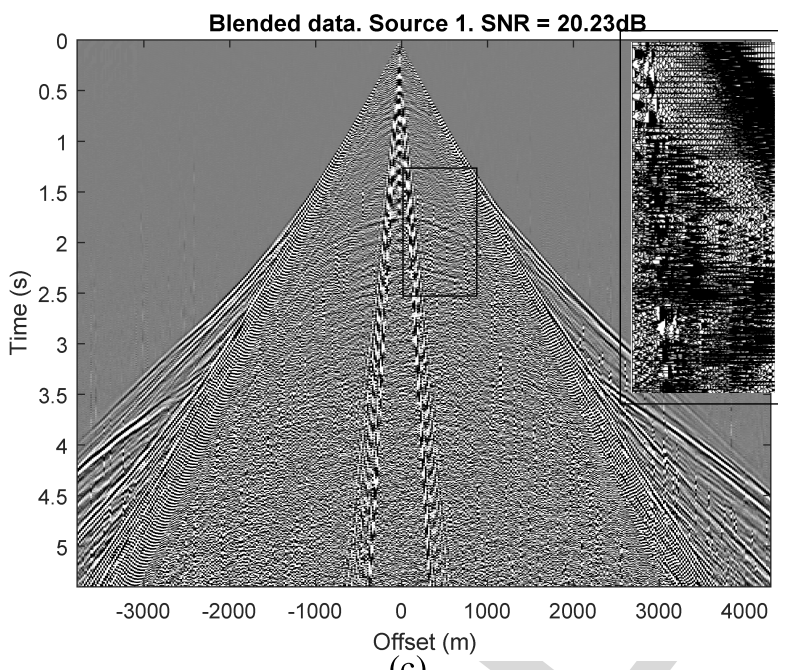

(c)

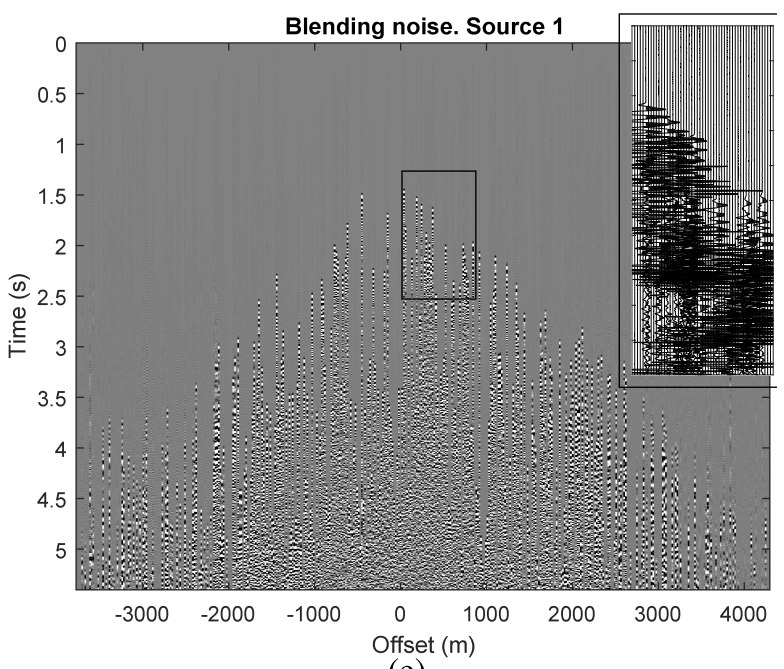

(e)
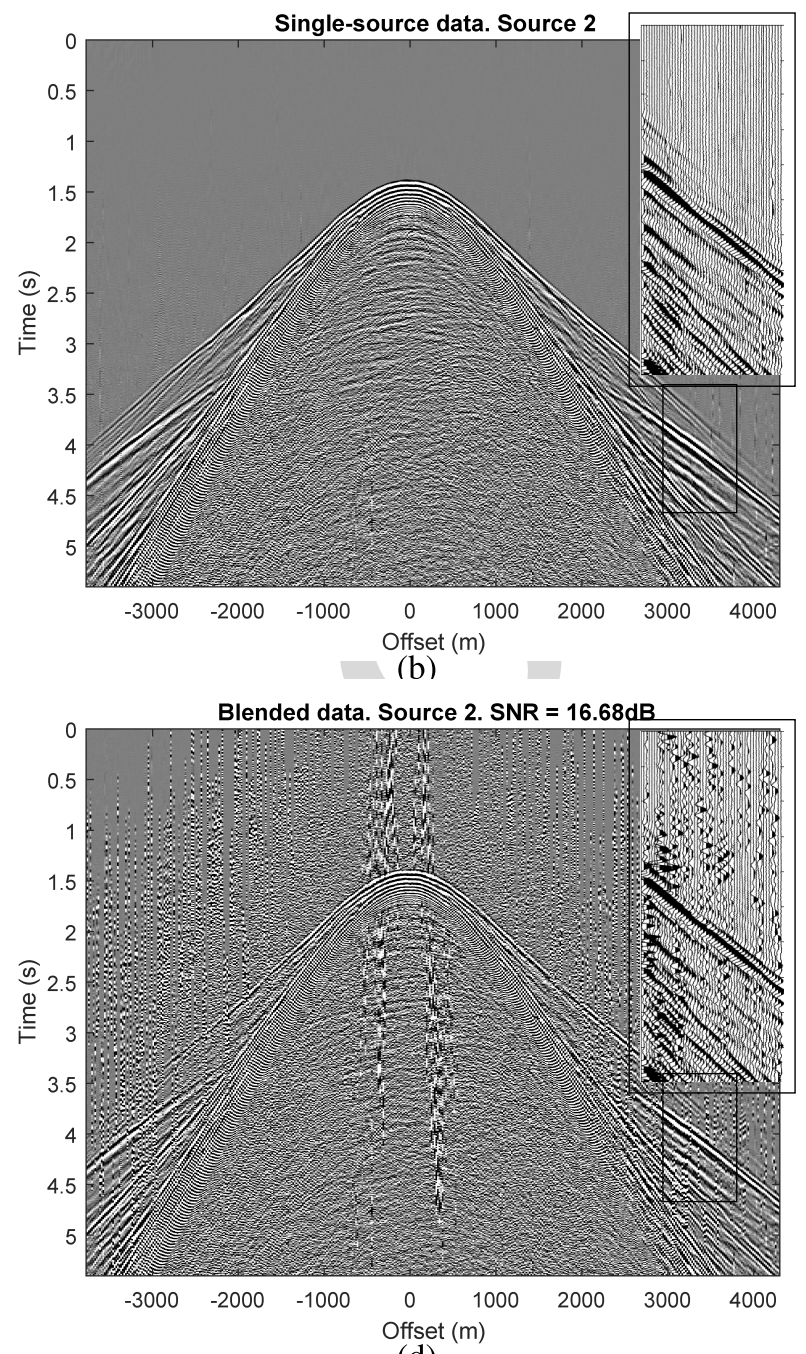

(d)

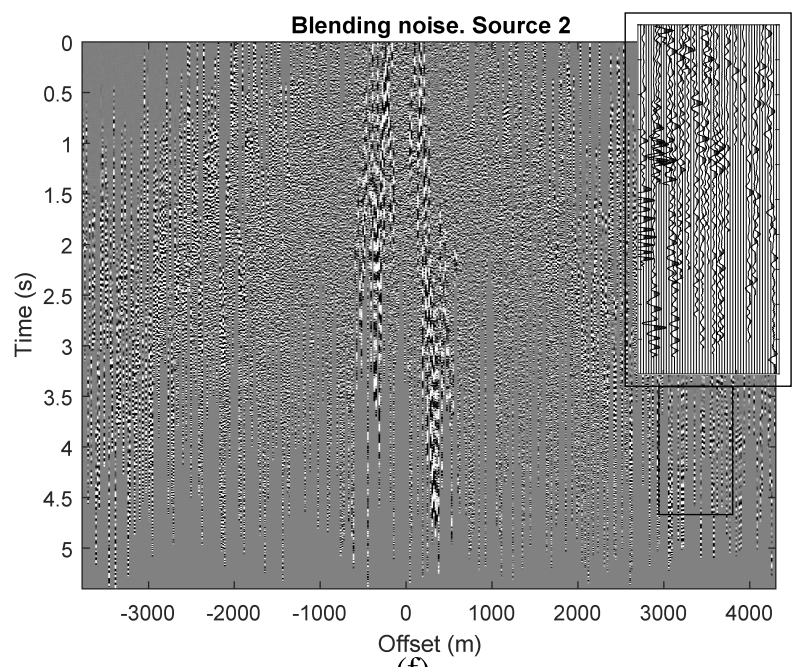

(f)

Fig. 10. (a) and (b) Real seismic data example: Torpille data. Input clean signal. (c) and (d) Artificially blended signal. (e) and (f) Isolated blending noise. For each image, its zoomed-in part highlighted by a rectangle is given at its top-right corner.

1) The OMP stopping criterion proposed in [45] is the achievement of a null, or at least of a sufficiently small $\ell_{2}$-norm of the residue $R^{L} d:\left\|R^{L} d\right\|<N_{R}$.
This approach is intuitive, but not easy to implement, as different seismic data sets do not have the same amplification, nor do they have the same level of ambient 


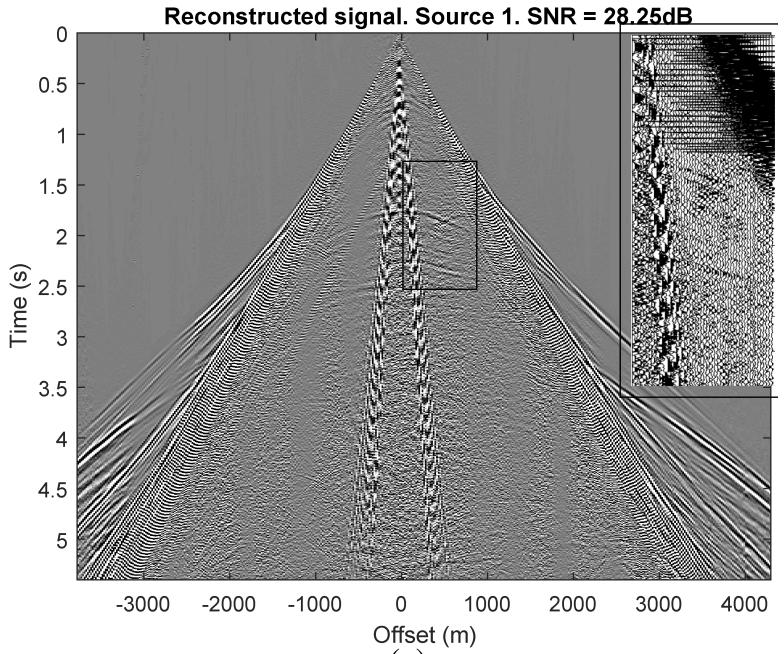

(a)

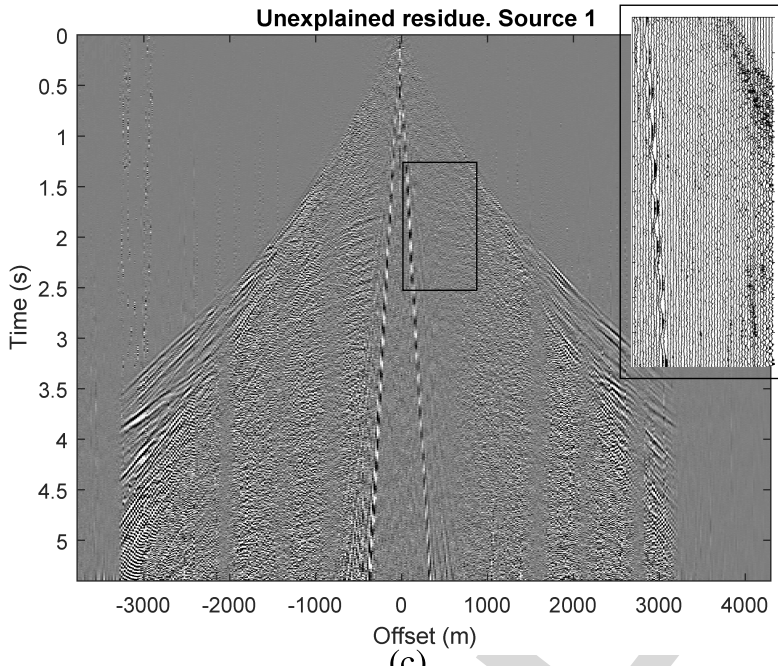

(c)

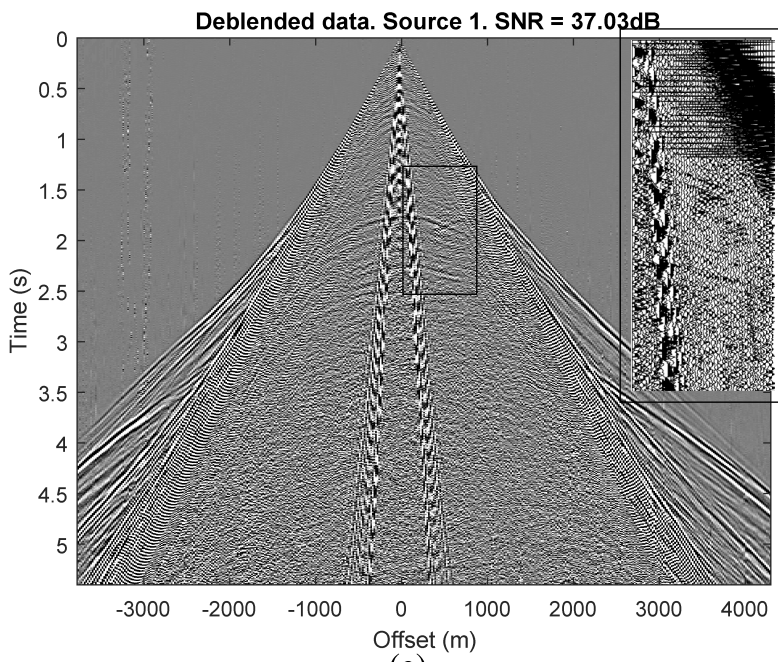

(e)
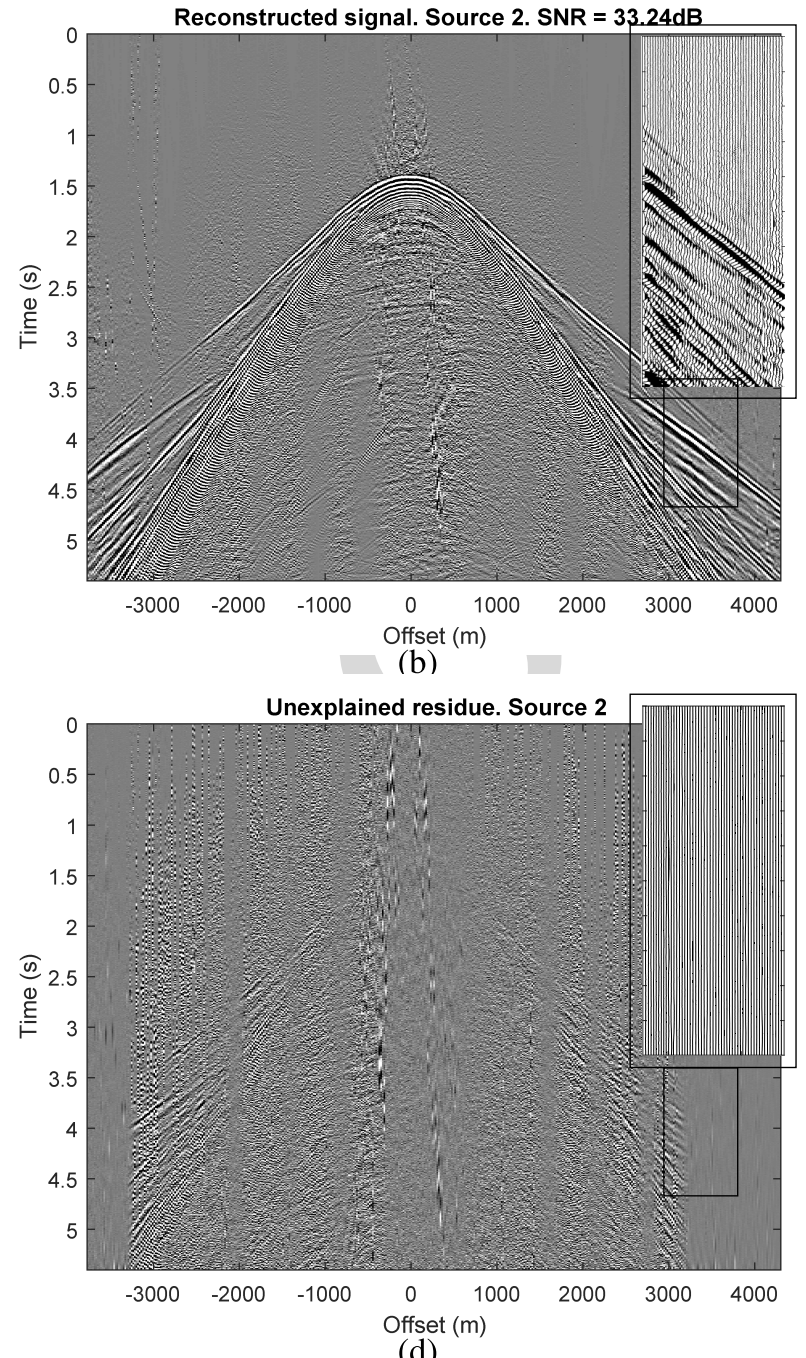

(d)

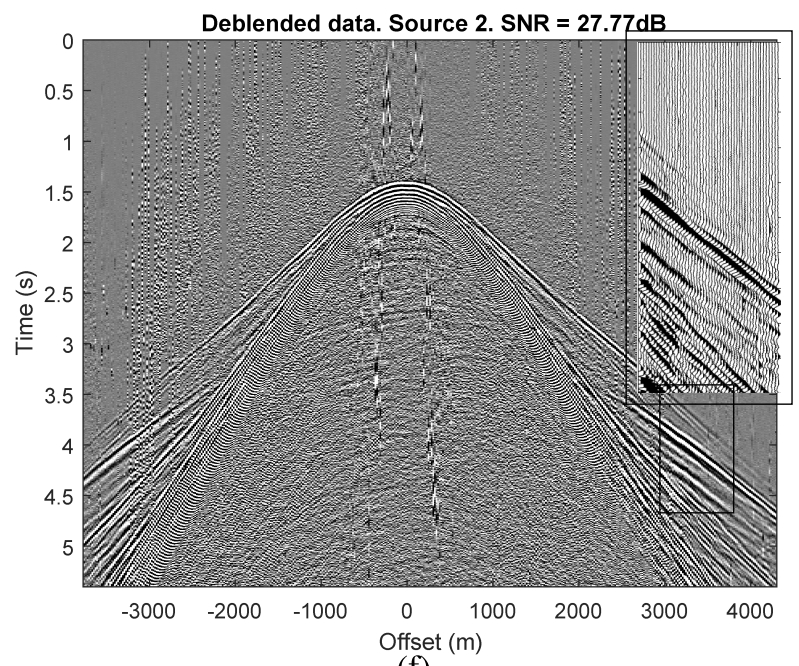

(f)

Fig. 11. (a) and (b) Explained signal. (c) and (d) Unexplained residue for the two sources after approximately 1000 iterations of OMP per lateral window. (e) and (f) Deblended data (explained signal of the source with the residue added) for the two sources. For each image, its zoomed-in part highlighted by a rectangle is given at its top-right corner.

noise or other noises which we would not want to reconstruct. In other words, the meta-parameter $N_{R}$ is difficult to choose as it is highly data dependent.
2) One relative value related to the residue energy is the relative residual energy $\left\|R^{L} d\right\|_{2}^{2} /\|\tilde{d}\|_{2}^{2}<E_{R}$. The metaparameter $E_{R}$ can easily be set to some very small value 
(of the order of computation error) in the absence of noise or can be derived from the pre-estimated signalto-noise ratio in the case of noisy data.

3) In some cases the parameters $N_{R}$ and even $E_{R}$ are difficult to define. If, in addition, the user only requires a low reconstruction precision (only wants to reconstruct and separate the most energetic events), it could be helpful to set $L_{\max }$ - the maximal number of iterations to perform-to a low value.

4) The reciprocal condition number is used to measure whether a matrix is well or badly conditioned (if this number is small). The condition number of a matrix affects the solutions of similar linear systems of equations: if the values of the matrix are slightly perturbed, this leads to big differences in the solution; thus, we stop the decomposition when this number is too small.

\section{REsults}

This section shows results obtained with the Matrioshka OMP algorithm, applied to a complex synthetic data set issued from the Marmousi geological model [60], with real seismic noise (see Section IV-A) and to real ocean bottom node (OBN) seismic data acquired in Torpille (Offshore Gabon) (see Section IV-B). More results on simple synthetic data examples that demonstrate the performance in "laboratory" conditions of the method can be found in [59].

\section{A. Complex Synthetics-Realistic Case Study}

We tested our method on a realistic data set, generated by the Allied Geophysical Laboratories of the University of Houston from the Marmousi geological model. Martin et al. [61] performed a highly precise elastic modeling to provide as many of the seismic features usually present in real seismic data as possible. Namely, the data contain not only primary and multiple reflections, but also diffractions, head waves, surface waves, scattering effects, and other realistic particularities. The acquisition geometry adopted for this simulation is that of a source vessel towing an airgun source at a depth of $10 \mathrm{~m}$ and performing a shot every $25 \mathrm{~m}$. The source signature is a zerophase 5-10-60-80 Hz Ormsby wavelet with frequencies up to $80 \mathrm{~Hz}$. The Ocean Bottom Cable is situated at the water bottom at a depth of $450 \mathrm{~m}$. We performed an artificial blending of the data by attributing different parts of the data to two different sources and creating overlapping shooting time patterns for each source: the first source shoots regularly, with an interval equal to $5 \mathrm{~s}$, and the second source shoots with irregular time intervals around $7 \pm 2 \mathrm{~s}$.

The first test, illustrated in Figs. 7 and 8, contains 20 traces for each source, which corresponds to a 500-m-wide lateral window. Fig. 7 shows decomposition residue and explained signal for the first source in the upper part of the section, where the signal is quite strong since it contains direct arrival and surface waves. Fig. 7(c) and (d) shows the decomposition result after only five iterations of the outer OMP: several of the most energetic seismic events have already been reconstructed, and the residue energy has significantly decreased. After 59 iterations of the outer OMP [see Fig. 7(e) and (f)], the useful signal present in the section is almost perfectly explained. The leakage of Source 1 remaining in the residue [see Fig. 7(f)] is present in the deblended signal associated with Source 2, but with a sufficiently low energy to be eliminated as acquisition noise by the classical downstream processing. However, because of the presence of significantly weaker signals in other parts of the studied sections, we continued the decomposition up to 1750 iterations of the outer OMP, getting a perfectly explained useful signal. The energy of the residue decreases almost linearly in logarithmic scale, as shown in Fig. 8(a). Fig. 8(b) shows the magnitude of the coefficients found during the decomposition. Note the rapid decrease in the beginning of the curve, indicating the sparsity of the transform. Fig. 8(c) and (d) shows for the two sources the increasing signal-tonoise ratio computed as $\mathrm{S} / \mathrm{N}=10 \log _{10}\left(\left\|d_{s}\right\|^{2} /\left\|d_{s}-d_{d}\right\|^{2}\right)$, where $d_{s}$ denotes the initial single source data, and $d_{d}$ the deblended data for the same source.

Fig. 9 shows a test on the same data, with the entire shot lines processed using sliding windows and in the presence of real seismic noise. Note that most of the noise is left in the residue, moreover to avoid any signal loss, the residue can be added back to the explained coherent events, if there is any signal left in it.

\section{B. Real Seismic Data Example}

In this section, we present test results on real data extracted from a 3-D OBN seismic survey acquired in Torpille. The acquisition used a conventional single source mode, with an airgun seismic source towed at a 7-m depth with a shot-point interval of $50 \mathrm{~m}$. The water depth in this area varies from 25 to $35 \mathrm{~m}$, which implies the presence of Scholte waves making the data almost as difficult to process as onshore. The sampling period was of $3 \mathrm{~ms}$, and the listening time for each shot was of $5.4 \mathrm{~s}$. We blended them artificially as for the Marmousi data. The clean and blended input data are shown in Fig. 10(a)-(d). Note that the shooting line of the first source is significantly closer to the receiver, since the useful signal in Fig. 10(b) and (d) is located deeper (i.e., later in time) than that in Fig. 10(a) and (c). Obviously, the further away the source is from the receiver, the weaker its recorded signal is. Therefore, the blending appears more aggressive for the second source than for the first one, as shown in Fig. 10(e) and (f). The first source, however, is also significantly contaminated, especially in the part where useful signals, as the primary reflections, are present (below $2 \mathrm{~s}$ ). The decomposition allows us to reconstruct the most energetic physical events, such as the direct arrivals, the surface waves and the guided waves. A significant part of the reflections is also reconstructed, which is well seen in the zoomed-in parts of Fig. 11(a) and (b). However, part of the coherent signals stays in the residue [see Fig. 11(c) and (d)]. Nevertheless, in order to avoid leakage, the residue can be added back to the reconstructed events for each source, as shown in Fig. 11(e) and (f). Note that the decomposition and deblending results for the real seismic data have inferior quality compared to the synthetic data with real noise added. This can be explained as follows. First, the Torpille data contain a significant part of incoherent noise-which 
our algorithm is not trying to capture-and when we blend data, we sum up the ambient noise recorded at different times, leading to a less favorable situation than a true simultaneous sources acquisition. Second, the big difference in the energy of the two sources is also difficult to handle, as sometimes the energetic noise tends to be reconstructed as coherent signal. Nevertheless, we were able to achieve a significant improvement of the signal-to-noise ratio for the deblending results shown in Fig. 11(e) and (f): around 10-15 dB for both sources. Taking into account that the deblending takes place in the very beginning of the processing sequence, the residual blending noise is likely to be handled by further conventional denoising or other processing. Limitations of our method include potential high computational complexity when big data sets need to be processed with a high level of precision. We addressed this issue by implementing analytical derivation in the optimization routines and fast norm calculation, but further code optimizations may be needed to industrialize the algorithm. Our method provides both deblended signals and a sparse representation of seismic data with a given precision, which is beneficial for diverse seismic data processing problems.

\section{CONCLUSION}

In this article, we have proposed a new source-separation method applied to seismic data acquired in simultaneoussource mode. This method consists of two nested OMPs and is called Matrioshka OMP. We have proposed two mathematical models of sensor signals in simultaneous-source seismic surveys. These models are justified by nonrestrictive assumptions on the seismic survey and the simultaneous sources, which we have stated as hypotheses. Our data-driven seismic event model is based on features which are characterized by spatial coherence of wavelet signals. Precisely, a seismic event is a straight or slightly curved feature in the trace representation of the data with a specific wavelet sufficiently stable within a local spatial window, whose magnitude can linearly vary according to the offset. We have deduced from this model specific dictionaries adapted to raw seismic data without preprocessing, and we have implemented two nested OMPs with these dictionaries. For this, we have efficiently solved a nonconvex optimization problem thanks to the gradual construction of the initial conditions close to the globally optimal solution. Finally, we have tested our method on complex synthetic seismic data with real noise and on real data. The synthetic data examples presented show excellent deblending results: the algorithm is capable of explaining almost all of the coherent seismic events present in the data. The real data example was more difficult to process, but the final results are acceptable in terms of further processing.

\section{ACKNOWLEDGMENT}

The authors would like to thank the anonymous reviewers for their comments that helped improve the presentation of this article.

\section{REFERENCES}

[1] W. Lynn, M. Doyle, K. Larner, and R. Marschall, "Experimantal investigation of interference from other seismic crews," Geophysics, vol. 52, pp. 1501-1524, 1987.

[2] P. I. Pecholcs et al., "Over 40,000 vibrator points per day with realtime quality control: Opportunities and challenges," in Proc. SEG Tech. Program Expanded Abstr., 2010, pp. 111-115.

[3] J. Kommedal, G. Alexander, L. Wyman, and S. Wagner, "ISS on ice: Seismic acquisition in the arctic," in Proc. SEG Tech. Program Expanded Abstr., 2016, pp. 6-10.

[4] M. G. Barbier and J. R. Viallix, "SOSIE: A new tool for marine seismology," Geophysics, vol. 38, no. 4, pp. 673-683, 1973.

[5] D. Silverman, "Method of three dimensional seismic prospecting," U.S. Patent 4159463 A, Jun. 26, 1979.

[6] H. J. Rozemond, "Slip-sweep acquisition," in Proc. SEG Tech. Program Expanded Abstr., 1996, pp. 64-67.

[7] H. Liu and R. Abma, "Simultaneous sources and deblending using multiple sweeps," in Proc. SEG Tech. Program Expanded Abstr., 2017, pp. 141-145.

[8] N. Moldoveanu, P. Jones, S. Totten, and E. Rosso, "Vibroseis simultaneous shooting using encoded sweeps: A field experiment," in Proc. SEG Tech. Program Expanded Abstr., 2017, pp. 146-150.

[9] A. Zhukov, I. Korotkov, E. Sidenko, I. Nekrasov, P. Gridin, and T. Galikeev, "Simultaneous pseudo-random shuffle-sweep generation and increased seismic data acquisition productivity," in Proc. SEG Tech. Program Expanded Abstr., 2017, pp. 151-155.

[10] C. J. Beasley, R. E. Chambers, and Z. Jiang, "A new look at simultaneous sources," in Proc. SEG Tech. Program Expanded Abstr., 1998, pp. 133135.

[11] S. T. Vaage, "Method and system for acquiring marine seismic data using multiple seismic sources," U.S. Patent 6906981 B2, Jun. 14, 2005.

[12] D. Howe, "Independent simultaneous sweeping-A method to increase productivity of land seismic crews," in Proc. SEG Tech. Program Expanded Abstr., 2008, pp. 2826-2830.

[13] G. Hampson, J. Stefani, and F. Herkenhoff, "Acquisition using simultaneous sources," Lead. Edge, vol. 27, no. 7, pp. 918-923, 2008.

[14] A. J. Berkhout, G. Blaquiere, and D. J. Verschuur, "From simultaneous shooting to blended acquisition," in Proc. SEG Tech. Program Expanded Abstr., 2008, pp. 2831-2838.

[15] R. Abma, Q. Zhang, A. Arogunmati, and G. Beaudoin, "An overview of BP's marine independent simultaneous source field trials," in Proc. SEG Tech. Program Expanded Abstr., 2012, pp. 1-5.

[16] W. Dai, X. Wang, and G. T. Schuster, "Least-squares migration of multisource data with a deblurring filter," Geophysics, vol. 76, no. 5, pp. R135-R146, 2011.

[17] D. J. Verschuur and A. J. Berkhout, "Seismic migration of blended shot records with surface-related multiple scattering," Geophysics, vol. 76 , no. 1, pp. A7-A13, 2011

[18] G. Henin et al., "Deblending 4-component simultaneous-source data-A 2D OBC case study in Malaysia," in Proc. SEG Tech. Program Expanded Abstr., 2015, pp. 43-47.

[19] P. Paramo, K. Vincent, A. Cegna, J. Kommedal, P. Napier, and S. Cardinez, "AVO analysis of independent simultaneous source OBC data from trinidad," in Proc. SEG Tech. Program Expanded Abstr., 2013, pp. 368-372.

[20] E. Shipilova et al., "Simultaneous-source seismic acquisitions: Do they allow reservoir characterization? A feasibility study with blended onshore real data," in Proc. SEG Tech. Program Expanded Abstr., 2016, pp. 107-112.

[21] T. Krupovnickas, K. Matson, C. Corcoran, and R. Pascual, "Marine simultaneous source OBS survey suitability for 4D analysis," in Proc. SEG Tech. Program Expanded Abstr., 2012, pp. $1-5$.

[22] D. M. Davies and M. Ibram, "Evaluating the impact of ISS HDOBC acquisition on 4D data," in Proc. 77th EAGE Conf. Exhib., 2015, pp. $1-5$.

[23] R. R. Haacke, G. Hampson, and B. Golebiowski, "Simultaneous shooting for sparse OBN 4D surveys and deblending using modified Radon operators," in Proc. 77th EAGE Conf. Exhib., 2015.

[24] K. Eggenberger et al., "Signal apparition-enabled parallel-source acquisition of 4D-grade seismic data: Results from a field test in the North Sea," in Proc. SEG Tech. Program Expanded Abstr., 2017, pp. 68-73. 
[25] I. Moore, "Removing seismic interference using simultaneous or near simultaneous source separation," U.S. Patent 0097885 A1, Apr. 22, 2010.

[26] J. Robertsson, L. Amundsen, and A. Pedersen, "Signal apparition for simultaneous source wavefield separation," Geophys. J. Int., vol. 206, no. 2, pp. 1301-1305, 2016.

[27] Z. Tang and X. Campman, "A coherent simultaneous shooting scheme and its source separation," in Proc. 78th EAGE Conf. Exhib., 2016.

[28] S. Huo, Y. Luo, and P. G. Kelamis, "Simultaneous sources separation via multidirectional vector-median filtering," Geophysics, vol. 77, no. 4, pp. V123-V131, 2012.

[29] C. Peng, B. Liu, A. Khalil, and G. Poole, "Deblending of simulated simultaneous sources using an iterative approach: An experiment with variable-depth streamer data," in Proc. SEG Tech. Program Expanded Abstr., 2013, pp. 4278-4282.

[30] S. Gan, Y. Wang, S. Chen, and X. Chen, "Deblending using a structuraloriented median filter," in Proc. SEG Tech. Program Expanded Abstr., 2015.

[31] S. Spitz, G. Hampson, and A. Pica, "Simultaneous source separation: A prediction-subtraction approach," in Proc. SEG Tech. Program Expanded Abstr., 2008, pp. 2811-2815.

[32] Z. Zhang, Q. Liu, Y. Xuan, H. Sun, Y. Hu, and L. Han, "The direct arrival in blended data," in Proc. SEG Tech. Program Expanded Abstr., 2016, pp. 275-279.

[33] C. Bagaini, M. Daly, and I. Moore, "The acquisition and processing of dithered slip-sweep vibroseis data," Geophys. Prospecting, vol. 60, no. 4, pp. 618-639, 2012.

[34] R. Abma and J. Yan, "Separating simultaneous sources by inversion," in Proc. 71st EAGE Conf. Exhib., 2009.

[35] R. Abma, T. Manning, M. Tanis, J. Yu, and M. Foster, "High-quality separation of simultaneous sources by sparse inversion," in Proc. 72nd EAGE Conf. Exhib., 2010.

[36] K. Wapenaar, J. van der Neut, and J. Thorbecke, "On the relation between seismic interferometry and the simultaneous-source method," Geophys. Prospecting, vol. 60, no. 4, pp. 802-823, 2012.

[37] R. Abma et al., "Independent simultaneous source acquisition and processing," Geophysics, vol. 80, no. 6, pp. WD37-WD44, 2015.

[38] I. Moore, "Simultaneous sources-Processing and applications," in Proc. 72nd EAGE Conf. Exhib., 2010.

[39] H. Mansour, H. Wason, T. Lin, and F. J. Herrmann, "Randomized marine acquisition with compressive sampling matrices," Geophys. Prospecting, vol. 60, no. 4, pp. 648-662, 2012.

[40] Y. Chen, "Deblending by iterative orthogonalization and seislet thresholding," in Proc. SEG Tech. Program Expanded Abstr., 2015, pp. 53-58.

[41] P. Doulgeris, A. Mahdad, and G. Blacquiere, "Iterative separation of blended marine data: Discussion on the coherencypass filter," in Proc. SEG Tech. Program Expanded Abstr., 2011, pp. 26-31.

[42] A. Mahdad, P. Doulgeris, and G. Blacquiere, "Separation of blended data by iterative estimation and subtraction of blending interference noise," Geophysics, vol. 76, no. 3, pp. Q9-Q17, 2011.

[43] P. Doulgeris, "Inversion methods for the separation of blended data," Ph.D. dissertation, Dept. Geophys., Delft Univ. Technol., Delft, The Netherlands, 2013.

[44] A. J. Berkhout, "Changing the mindset in seismic data acquisition," Lead. Edge, vol. 27, pp. 924-938, Jul. 2008.

[45] S. G. Mallat and Z. Zhang, "Matching pursuits with timefrequency dictionaries," IEEE Trans. Signal Process., vol. 41, no. 12, pp. 3397-3415, Dec. 1993

[46] Y. C. Pati, R. Rezaiifar, and P. S. Krishnaprasad, "Orthogonal matching pursuit: Recursive function approximation with applications to wavelet decomposition," in Proc. 27th Asilomar Conf. Signals, Syst. Comput., Nov. 1993, pp. 40-44.

[47] T. Nguyen and J. Castagna, "Matching pursuit of two dimensional seismic data and its filtering application," in Proc. SEG Tech. Program Expanded Abstr., 2000.

[48] P. Hugonnet and J.-L. Boelle, "Beyond aliasing regularisation by plane event extraction," in Proc. 69th EAGE Conf. Exhib., 2007.

[49] P. Hugonnet, J.-L. Boelle, and F. Prat, "Local linear events extraction and filtering in the presence of time-shifts," in Proc. 74th EAGE Conf. Exhib., 2012.

[50] J. Wang, M. Ng, and M. Perz, "Seismic data interpolation by greedy local Radon transform," Geophysics, vol. 75, no. 6, pp. WB225-WB234, 2010 .

[51] A. Adamo, P. Mazzucchelli, and N. Bienati, "Weak orthogonal matching pursuit with geophysical atom selection," in Proc. 76th EAGE Conf. Exhib., 2014.

[52] F. Boßmann and J. Ma, "Asymmetric chirplet transform for sparse representation of seismic data," Geophysics, vol. 80, no. 6 , pp. WD89-WD100, 2015.

[53] H. Hu, Y. Liu, A. Osen, and Y. Zheng, "Compression of local slant stacks by the estimation of multiple local slopes and the matching pursuit decomposition," Geophysics, vol. 80, no. 6, pp. WD175-WD187, 2015

[54] R. Zhang and J. Castagna, "Seismic sparse-layer reflectivity inversion using basis pursuit decomposition," Geophysics, vol. 76, no. 6 , pp. R147-R158, 2011.

[55] R. E. Sheriff and L. P. Geldart, Exploration Seismology, 2nd ed. Cambridge, U.K.: Cambridge Univ. Press, 1995.

[56] D. Hampson, "Inverse velocity stacking for multiple elimination," J. Can. Soc. Explor. Geophys., vol. 22, no. 1, pp. 44-55, 1986.

[57] P. L. Stoffa, J. B. Diebold, and P. Buhl, "Inversion of seismic data in the $\tau$-p plane," Geophys. Res. Lett., vol. 8, no. 8, pp. 869-872, 1981.

[58] E. Shipilova, J.-L. Boelle, M. Bloch, M. Barret, and J.-L. Collette, "Matrioshka orthogonal matching pursuit for blended seismic source separation," in Proc. SEG Tech. Program Expanded Abstr., 2017, pp. 4919-4924.

[59] E. Shipilova, "Separation of signals originating from simultaneous seismic sources by greedy signal decomposition methods," Ph.D. dissertation, Dept. Signal Process., CentraleSupélec, France, 2018.

[60] R. Versteeg, "The Marmousi experience: Velocity model determination on a synthetic complex data set," Lead. Edge, vol. 13, no. 9, pp. 927-936, Sep. 1994.

[61] G. S. Martin, R. Wiley, and K. J. Marfurt, "Marmousi2: An elastic upgrade for Marmousi," Lead. Edge, vol. 25, no. 2, pp. 156-166, Jan. 2006

1087

1088 1089

1090 1091

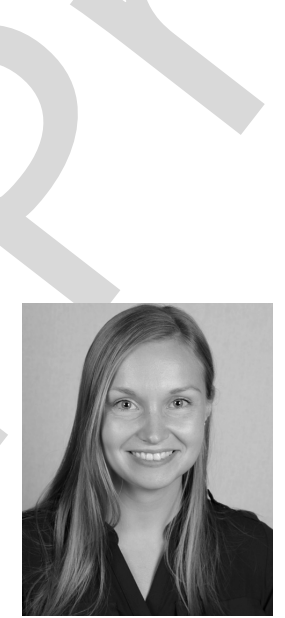

Ekaterina Shipilova received the bachelor's and master's degrees in geology from Lomonosov Moscow State University, Moscow, Russia, in 2011 and 2013, respectively, and the Ph.D. degree in signal processing from CentraleSupélec, Metz, France, in 2018.

In 2013, she joined TOTAL, Pau, France, to work as a Research Geophysicist in seismic acquisition and processing. She moved to Paris in 2018 to join the Total's Petroleum Basins Evaluation Department, where she is currently working as a Synthesis Geoscientist. Her research interests include seismic acquisition and processing, seismic attributes, and seismic characterization of source rocks. 1119 1120 1121 1122 1123 1124 1125 1126 1127 1128 1129 1130

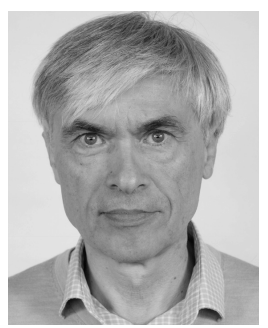

Michel Barret received the Engineering degree from Ecole Supélec, Paris, France, the Ph.D. and Habilitation degrees in signal processing from the University of Paris-Sud, Orsay, France, in 1993 and 2010, respectively.

In 1986, he joined the Automatics and Signa Processing Department, Ecole Supélec, where he became an Associate Professor in 1997 and a Professor in 2011. He is currently a Professor with CentraleSupélec, Metz, France, where he teaches statistical signal processing. He is also with the UMI 2958 Georgia Tech-CNRS, Metz. His research interests include stability of multidimensional digital recursive filters, adapted filter banks, multicomponent image compression, statistical processes, and signal representations. 


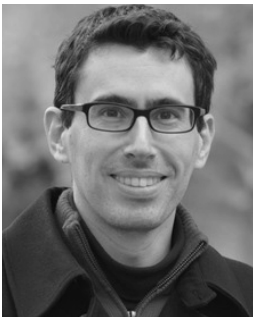

Matthieu Bloch received the Engineering degree from Supélec, Gif-sur-Yvette, France, in 2003, the M.S. degree in electrical engineering from the Georgia Institute of Technology, Atlanta, GA, USA, in 2003, the Ph.D. degree in engineering science from the Université de Franche-Comté, Besançon, France, in 2006, and the Ph.D. degree in electrical engineering from the Georgia Institute of Technology (Georgia Tech.) in 2008.

From 2008 to 2009, he was a Post-Doctoral Research Associate with the University of Notre Dame, South Bend, IN, USA. Since July 2009, he has been with the Faculty of the School of Electrical and Computer Engineering. From 2009 to 2013, he was based at Georgia Tech Lorraine, Metz, France. He is currently an Associate Professor with the School of Electrical and Computer Engineering, Georgia Tech. He is the coauthor of the textbook Physical-Layer Security: From Information Theory to Security Engineering (Cambridge University Press). His research interests are in the areas of information theory, errorcontrol coding, wireless communications, and cryptography.

Dr. Bloch was a co-recipient of the IEEE Communications Society and the IEEE Information Theory Society 2011 Joint Paper Award. He has served on the organizing committee of several international conferences. He was the Chair of the Online Committee of the IEEE Information Theory Society from 2011 to 2014, an Associate Editor of the IEEE TRANSACTIONS ON INFORMATION from 2016 to 2019, and he has been on the Board of Governors of the IEEE Information Theory Society. He has been an Associate Editor of the IEEE TRANSACTIONS ON INFORMation ForEnsics AND SECURITY since 2016.

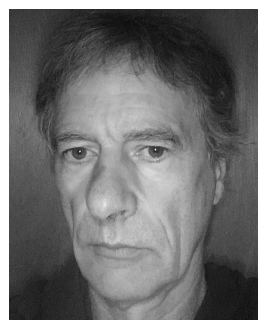

Jean-Luc Boelle received the Engineering degree from École Centrale des Arts et Manufactures, Paris, France, in 1977, and the Ph.D. degree from the Soils and Structures Laboratory, Ecole Centrale Paris, Paris, in 1983.

He joined the French Research Institute for Ocean Science, Brest, France, in 1983 and TOTAL, Pau, France, Oil and Gas company, in 1988. He worked and headed several research projects dealing with seismic wave propagation modeling, and seismic data acquisition and processing.

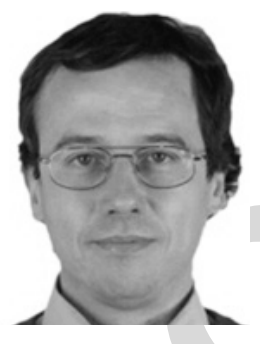

Jean-Luc Collette received the Engineering degree from Ecole Supélec, Paris, France, in 1985.

In 1986, he joined the Automatics and Signal Processing Department, Ecole Supélec. He is currently a Professor with CentraleSupélec, Metz, France, where he teaches automatic control and image processing. His research interests include hybrid filter banks and biomedical imaging. 